\title{
ON THE CLASSIFICATION OF NONCOMPACT COMPLEX ABELIAN LIE GROUPS( $\left.{ }^{(}\right)$
}

\author{
BY \\ AKIHIKO MORIMOTO
}

1. Introduction. The purpose of this paper is to investigate the structures of some types of noncompact complex abelian Lie groups and to classify all noncompact connected complex abelian Lie groups of dimension 3. In a previous paper [5] we have constructed a complex Lie group, on which every holomorphic function is a constant and which contains no complex torus of positive dimension. Such a group was called an (H.C)-group. We have then characterized noncompact complex Lie group of dimension two to be an (H.C)-group (Theorem 5 of [5]). Furthermore we have classified all connected complex abelian Lie groups of dimension 2.

In this paper we first prove that any connected complex abelian Lie group is isomorphic to the product group of an (H.C)-group and a group which is a Stein manifold (\$3).

In $\$ 4$ we generalize the theorem mentioned above and we shall characterize a complex Lie group of arbitrary dimension to be an (H.C)-group.

In $\$ 5$ we shall consider $n$-dimensional connected complex abelian Lie groups $G$ of rank $n+1$, namely $G$ is the factor group of $C^{n}$ by a discrete subgroup $\Gamma$ which is generated by $n+1$ vectors of $C^{n}$ linearly independent over the real number field and contains $n$ vectors linearly independent over the complex number field. The family $A(n)$ of such groups will be classified into two subfamilies - one is the family of (H.C)-groups and the other is the family $A(n-1) \times\left\{C^{*}\right\}$, $C^{*}$ being the multiplicative group of nonzero complex numbers.

In $\$ 6$ we shall prove that a complex abelian Lie group containing no complex torus of positive dimension contains no compact complex submanifold of positive dimension, and we shall remark that the group constructed in Theorem 3 [5] does not contain any compact complex submanifold of positive dimension.

In $\S \S 7,8$ we shall introduce the notion of nonsingular matrices of $\boldsymbol{n}$ rows and $m$ columns for $n>m$ and the one of nonsingular abelian Lie groups of dimension $n$ and of rank $n+m$. In the final part of $\S 8$ we shall classify the family of all nonsingular complex abelian Lie groups of arbitrary dimension. Nonsingular complex abelian Lie groups contain no complex torus of positive dimension.

Received by the editors May 28, 1965.

(1) This research was supported by the Air Force Office of Scientific Research. 
However, the converse of this statement is not always true, which causes complications in the classification of complex abelian Lie groups.

In \$§9-11 we consider abelian Lie groups of dimension 3 and of rank 5. In fact, in $\$ 10$ we classify singular groups containing no complex torus and in $\S 11$ we classify (H.C)-groups of dimension 3 containing a complex torus of 1 dimension not as a direct summand.

In $\$ 12$, summarizing the results obtained in the preceding sections we can classify all connected noncompact complex abelian Lie groups of dimension 3 . In summary, we may say that, in a sense, most of noncompact complex abelian Lie group $G$ is a Lie group containing no compact complex submanifold such that every holomorphic function on $G$ is necessarily a constant.

In the sequel, we shall denote, as usual, by $\boldsymbol{C}, \boldsymbol{R}, \boldsymbol{Q}$ and $\boldsymbol{Z}$ the ring of all complex numbers, real numbers, rational numbers and rational integers respectively.

2. Preliminary lemmas. In this section we shall recall some results in [5] and prove some preliminary lemmas which will be used later.

A complex Lie group $G$ will be called a Stein group if $G$ is a Stein manifold as a complex manifold. $G$ will be called an (H.C)-group if every holomorphic function on $G$ is a constant. Every connected complex Lie group $G$ contains uniquely a closed connected complex normal subgroup $G^{0}$ such that the factor group $G / G^{0}$ is a Stein group and that $G^{0}$ is an (H.C)-group. Every (H.C)-group is abelian.

Let $G$ be an $n$-dimensional connected complex abelian Lie group. Then $G$ is isomorphic to the factor group $\boldsymbol{C}^{n} / \Gamma$ of $\boldsymbol{C}^{n}$ by a discrete subgroup $\Gamma$ of $\boldsymbol{C}^{n}$. The group $\Gamma$ is generated by $u_{1}, \cdots, u_{k}$ which are linearly independent over $\boldsymbol{R}$. The number $k$ will be called the rank of $G$.

Definition 2.1. Let $P$ be one of the rings $C, R, Q$ and $Z$. We denote by $\mathfrak{M}(n, m ; P)$ the $P$-module of all matrices of $n$ rows and $m$ columns with coefficients in $P$, where $n, m \geqq 1$. We identify $P^{n}$ with $\mathfrak{M}(n, 1 ; P)$. For any $M \in \mathfrak{M}(n, m ; P)$ we denote by ${ }^{t} M \in \mathfrak{M}(m, n ; P)$ the transposed matrix of $M$. We write $\mathfrak{M}(n, n ; P)=\mathfrak{M}(n, P)$ and the group of all element $M \in \mathfrak{M}(n, P)$ with nonvanishing determinant, $\operatorname{det} M \neq 0$ (in the case $P=Z$, $\operatorname{det} M= \pm 1$ ) will be denoted by $\operatorname{GL}(n, P)$. For $M \in \mathfrak{M}(n, m ; P)$ and $N \in \mathfrak{M}(n, l ; P)$ we denote by $(M, N)(\in \mathbb{M}(n, m+l ; P))$ the matrix obtained by arranging $M$ as the first $m$ columns and $N$ as the last $l$ columns.

We denote by $E_{n}$ the unit matrix of degree $n$.

For $n>m$, we denote by $\mathfrak{M}^{*}(n, m ; C)$ the set of all $V \in \mathfrak{M}(n, m ; C)$ such that $e_{1}, e_{2}, \cdots, e_{n}, v_{1}, \cdots, v_{m}$ are linearly independent over $\boldsymbol{R}$, where $e_{i}$ is the $i$ th unit vector of $C^{n}$ and where we have put $V=\left(v_{1}, \cdots, v_{m}\right)$ with $v_{i} \in C^{n}(i=1,2, \cdots, n)$. We remark that $\mathfrak{M}^{*}(n, 1 ; C)=C^{n}-\boldsymbol{R}^{n}$ holds. For any $V \in \mathfrak{M} *(n, m ; C)$ we denote by $\Gamma(V)$ the discrete subgroup of $C^{n}$ generated by $e_{1}, \cdots, e_{n}, v_{1}, \cdots, v_{m}$. 
For any elements $u_{1}, \cdots, u_{k} \in P^{n}$ we denote by $\left\{u_{1}, \cdots, u_{k}\right\}_{P}$ the set of all elements $\sum_{i=1}^{k} c_{i} u_{i}$ with $c_{i} \in P(i=1, \cdots, k)$.

LEMMA 2.2. Let

$$
M=\left(\begin{array}{ll}
A & C \\
B & D
\end{array}\right) \in \mathrm{GL}(n+m, Z)
$$

with $A \in \mathfrak{M}(n, Z)$ and let $V \in \mathfrak{M}(n, m ; C)$. Supposc there exists $V^{\prime} \in \mathfrak{M}(n, m ; C)$ such that

$$
(A+V B) V^{\prime}=C+V D .
$$

Then $\operatorname{det}(A+V B) \neq 0$. Moreover, if $n>m$ and $V^{\prime} \in \mathfrak{M}^{*}(n, m ; C)$, then $V \in \mathfrak{M}^{*}(n, m ; C)$.

Proof. Put $\phi=A+V B$. Then, $\phi\left(E_{n}, V^{\prime}\right)=\left(A+V B,(A+V B) V^{\prime}\right)$ $=(A+V B, C+V D)=\left(E_{n}, V\right) M$. Hence we have $\phi\left(E_{n}, V^{\prime}\right) M^{-1}=\left(E_{n}, V\right)$. Putting $\left(E_{n}, V^{\prime}\right) M^{-1}=\left(u_{1}, u_{2}, \cdots, u_{n+m}\right)$ with $u_{i} \in C^{n}$, we have $\phi\left(u_{i}\right)=e_{i}(i=1,2, \cdots, n)$. Hence $\operatorname{det} \phi \neq 0$. Further, if $V^{\prime} \in \mathfrak{M}^{*}(n, m ; C)$, we assert that $u_{1}, \cdots, u_{n+m}$ are linearly independent over $\boldsymbol{R}$. In fact, if $\sum r_{i} u_{i}=0$ for $0 \neq \neq^{t}\left(r_{1}, \cdots, r_{n+m}\right) \in \boldsymbol{R}^{n+m}$, put ${ }^{t}\left(r_{1}^{\prime}, \cdots, r_{n+m}^{\prime}\right)=M^{-1} \cdot{ }^{t}\left(r_{1}, \cdots, r_{n+m}\right)$. Then the equality $\left(E_{n}, V^{\prime}\right) \cdot{ }^{t}\left(r_{1}^{\prime}, \cdots, r_{n+m}^{\prime}\right)$ $=\left(u_{1}, \cdots, u_{n+m}\right) \cdot M \cdot M^{-1} \cdot{ }^{t}\left(r_{1}, \cdots, r_{n+m}\right)=0$ means that $e_{1}, \cdots, e_{n}, v_{1}^{\prime}, \cdots, v_{m}^{\prime}$ are linearly dependent over $R$, where we have put $V^{\prime}=\left(v_{1}^{\prime}, \cdots, v_{m}^{\prime}\right)$. Hence our assertion is proved. Since $u_{1}, \cdots, u_{n+m}$ are linearly independent over $\boldsymbol{R}$ and since $\left(\phi\left(u_{1}\right), \cdots, \phi\left(u_{n+m}\right)\right)=\left(E_{n}, V\right), e_{1}, \cdots, e_{n}, v_{1}, \cdots, v_{m}$ are linearly independent over $\boldsymbol{R}$, where $V=\left(v_{1}, \cdots, v_{m}\right)$. Hence $\left.V \in \mathfrak{M}\right)^{*}(n, m ; C)$. Thus Lemma 2.2 is proved.

LEMMA 2.3. Let

$$
M=\left(\begin{array}{ll}
A & C \\
B & D
\end{array}\right) \in \mathrm{GL}(n+m, Z)
$$

with $A \in \mathfrak{M}(n, Z)$ and $V, V^{\prime} \in \mathfrak{M}(n, m ; C)$. Put

$$
M^{-1}=\left(\begin{array}{ll}
A^{\prime} & C^{\prime} \\
B^{\prime} & D^{\prime}
\end{array}\right)
$$

with $A^{\prime} \in \mathfrak{M}(n, Z)$. Then, $(A+V B) V^{\prime}=C+V D$ holds if and only if $\left(A^{\prime}+V^{\prime} B^{\prime}\right) V=C^{\prime}+V^{\prime} D^{\prime}$ holds.

Proof. Put $\phi=A+V B$. Suppose that $(A+V B) V^{\prime}=C+V D$ holds. Then, as is shown in the proof of Lemma 2.2, we have $\phi\left(E_{n}, V^{\prime}\right)=\left(E_{n}, V\right) M$. Hence we have $\left(E_{n}, V^{\prime}\right) M^{-1}=\phi^{-1}\left(E_{n}, V\right)=\left(\phi^{-1}, \phi^{-1} V\right)$. Thus we have $A^{\prime}+V^{\prime} B^{\prime}=\phi^{-1}$ and $C^{\prime}+V^{\prime} D^{\prime}=\phi^{-1} V$, which imply $C^{\prime}+V^{\prime} D^{\prime}=\left(A^{\prime}+V^{\prime} B^{\prime}\right) V$. The converse is clear from what we have proved. Thus Lemma 2.3 is proved.

LemmA 2.4. Take $V, V^{\prime} \in \mathfrak{M} *(n, m ; C)$. Then two complex Lie groups $C^{n} / \Gamma(V)$ and $\boldsymbol{C}^{n} / \Gamma\left(V^{\prime}\right)$ are (holomorphically) isomorphic, if and only if there exists a matrix $M \in \mathrm{GL}(n+m, Z)$ satisfying the following condition. 


$$
(A+V B) V^{\prime}=C+V D
$$

where

$$
M=\left(\begin{array}{ll}
A & C \\
B & D
\end{array}\right)
$$

with $A \in \mathfrak{M}(n, Z)$.

Proof. Suppose $\boldsymbol{C}^{n} / \Gamma(V) \simeq \boldsymbol{C}^{n} / \Gamma\left(V^{\prime}\right)$. Then the isomorphism $\tilde{\phi}$ of $\boldsymbol{C}^{n} / \Gamma(V)$ onto $\boldsymbol{C}^{n} / \Gamma\left(V^{\prime}\right)$ induces an automotphism $\phi$ of $\boldsymbol{C}^{n}$ such that $\phi(\Gamma(V))=\Gamma\left(V^{\prime}\right)$. Hence $\left\{\phi\left(e_{1}\right), \cdots, \phi\left(e_{n}\right), \phi\left(v_{1}\right), \cdots, \phi\left(v_{m}\right)\right\}_{z}=\left\{e_{1}, \cdots, e_{n}, v_{1}^{\prime}, \cdots, v_{m}^{\prime}\right\}_{z}$, where $V=\left(v_{1}, \cdots, v_{m}\right)$ and $V^{\prime}=\left(v_{1}^{\prime}, \cdots, v_{m}^{\prime}\right)$. Then, there exists a matrix $M=\left(a_{i j}\right) \in \mathfrak{M}(n+m, Z)$ such that

$$
\begin{aligned}
& e_{i}=\sum_{j=1}^{n} \phi\left(e_{j}\right) a_{j i}+\sum_{k=1}^{m} \phi\left(v_{k}\right) a_{n+k, i}, \\
& v_{k}^{\prime}=\sum_{j=1}^{n} \phi\left(e_{j}\right) a_{j, n+k}+\sum_{s=1}^{m} \phi\left(v_{s}\right) a_{n+s, n+k}
\end{aligned}
$$

for $i=1, \cdots, n ; k=1, \cdots, m$. We see readily that $M \in \mathrm{GL}(n+m, Z)$. Considering $\phi$ as an element $\operatorname{GL}(n, C)$ we can write $(2.2)$ as the following matrix form.

$$
\left(E_{n}, V^{\prime}\right)=\phi \cdot\left(E_{n}, V\right) \cdot M \text {. }
$$

The right-hand side of (2.3) is equal to $(\phi(A+V B), \phi(C+V D))$. Hence we have $E_{n}=\phi(A+V B)$ and $V^{\prime}=\phi(C+V D)$. Then, $\phi^{-1}=A+V B$ and $\phi^{-1} V^{\prime}=C+V D$ imply the equality (2.1).

Conversely, if $(2.1)$ holds, we see by Lemma 2.2 that $\operatorname{det}(A+V B) \neq 0$. Hence we can put $\phi=(A+V B)^{-1}$. Furthermore we see that (2.3) holds, which means the conditions (2.2). Therefore we have $\phi(\Gamma(V))=\Gamma\left(V^{\prime}\right)$. Then, $\phi$ induces an isomorphism of $\boldsymbol{C}^{n} / \Gamma(V)$ onto $\boldsymbol{C}^{n} / \Gamma\left(V^{\prime}\right)$. Thus Lemma 2.4 is proved.

We can easily prove the following:

Lemma 2.5. Let $V \in \mathfrak{M}^{*}(n, m ; C)$ and

$$
M=\left(\begin{array}{ll}
A & C \\
B & D
\end{array}\right) \in \mathrm{GL}(n+m, Z)
$$

with $A \in \mathfrak{M}(n, Z)$. Put $A+V \cdot B=\left(u_{1}, \cdots, u_{n+m}\right)$ with $u_{i} \in C^{n}(i=1, \cdots, n+m)$. Then $\Gamma(V)=\left\{u_{1}, \cdots, u_{n+m}\right\}_{Z}$ holds.

LEMMA 2.6. Suppose $a_{1}, a_{2}, \cdots, a_{n} \in Z(n \geqq 2)$ are coprime: $\left(a_{1}, a_{2}, \cdots, a_{n}\right)=1$ and $a_{1} \neq 0$. Then, there exists a matrix $M=\left(a_{i j}\right) \in \mathfrak{M}(n, Z)$ such that $a_{1 j}=a_{j}$ $(j=1,2, \cdots, n)$ and that $\operatorname{det} M=1$.

Proof. The matrix $M$ is constructed inductively as follows. Put $p_{1}=\left(a_{1}, \cdots, a_{n-1}\right)$ (greatest common divisor of $\left.a_{1}, \cdots, a_{n-1}\right), a_{k}=p_{1} \cdot a_{k}^{1} \quad(k=1, \cdots, n-1)$, 
$p_{2}=\left(a_{1}^{1}, a_{2}^{1}, \cdots, a_{n-2}^{1}\right), \quad a_{k}^{1}=p_{2} \cdot a_{k}^{2} \quad(k=1, \cdots, n-2), \quad p_{3}=\left(a_{1}^{2}, \cdots, a_{n-3}^{2}\right)$, $a_{k}^{2}=p_{3} \cdot a_{k}^{3}(k=1, \cdots, n-3)$ and so on. Then, since $\left(p_{i}, a_{n-i+1}^{i-1}\right)=1$, there exist $s_{i}, t_{i} \in Z$ such that $t_{i} p_{i}-s_{i} a_{n-i+1}^{i-1}=1$ for $i=1,2, \cdots, n-1$, where we have put $a_{n}^{0}=a_{n}$. Next, put $b_{i j}=s_{i} a_{j}^{i}$ for $j=1,2, \cdots, n-i ; i=1,2, \cdots, n-1$ and define $M$ as follows:

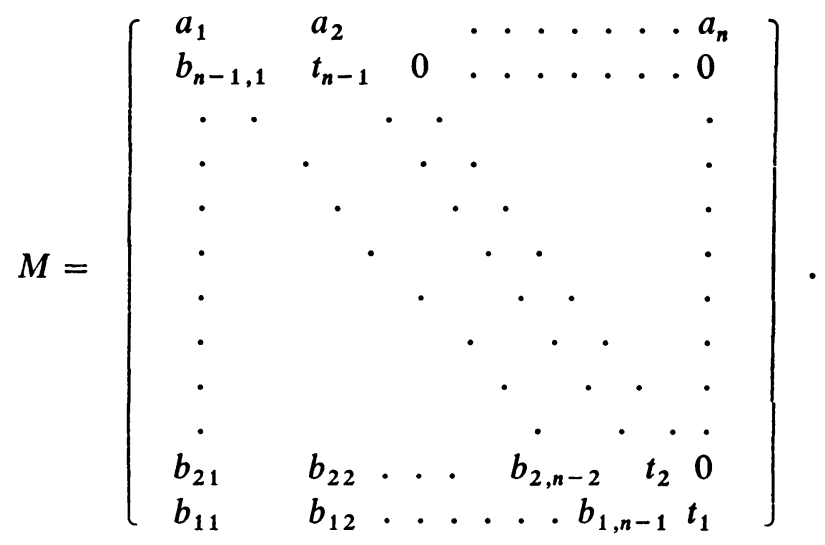

Now we prove that $\operatorname{det} M=1$ by induction on $n$. If $n=2$ the lemma is clearly true. Suppose that Lemma 2.6 is true for $n-1$. Then by expanding $\operatorname{det} M$ with respect to the $n$th column we have

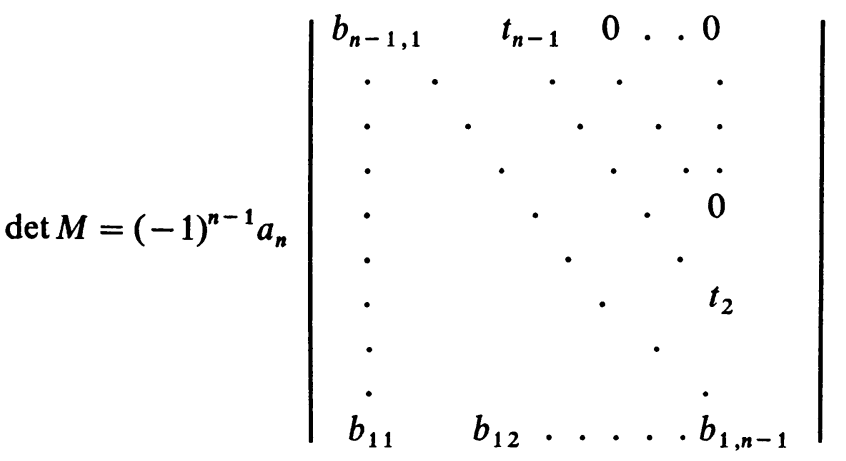

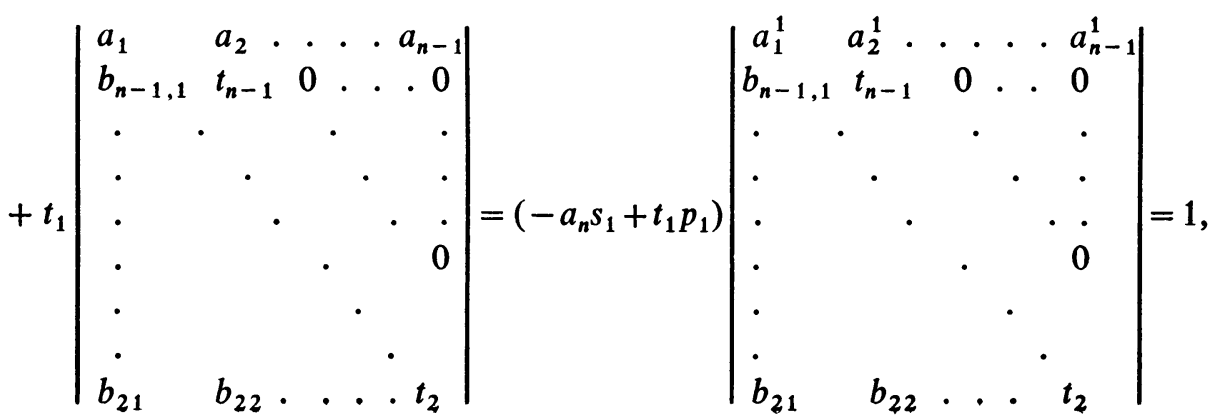


where we have used the induction assumption in the third equality. Thus Lemma 2.6 is proved.

3. Decomposition of complex abelian Lie groups. In this section we shall prove that any connected complex abelian Lie group is isomorphic to the direct product of an (H.C)-group and a Stein group (cf. [2]).

LEMma 3.1. Let $G$ be a connected complex abelian Lie group and $G^{\prime}$ be $a$ connected closed complex subgroup of $G$. If the factor group $G / G^{\prime}$ is isomorphic to $C$ (or $\left.C^{*}\right)$, then $G$ is isomorphic to the direct product $G^{\prime} \times C\left(\right.$ or $G^{\prime} \times C^{*}$ resp.).

Proof. We can suppose that $G=C^{n} / \Gamma$, where $\Gamma$ is a discrete subgroup of $C^{n}$. Let $\phi$ be the natural homomorphism of $C^{n}$ onto $G$. Let $W$ be the connected component of $\phi^{-1}\left(G^{\prime}\right)$ containing the unit element $e$ of $G$. The restriction $\phi_{1}=\phi / W$ of $\phi$ to $W$ defines a covering map of $W$ onto $G^{\prime}$. Hence $G^{\prime} \simeq W / \phi^{-1}(e)=W / W \cap \Gamma$. Hence $W+\Gamma / \Gamma=G^{\prime}$.

(1) Assume that $G / G^{\prime} \simeq C$.

Since $\left(C^{n} / W\right) /(W+\Gamma / W) \simeq C^{n} /(W+\Gamma) \simeq G / G^{\prime} \simeq C$, and since $C^{n} / W \simeq C$, we have $W+\Gamma=W$, whence $\Gamma \subset W$ and so $W \cap \Gamma=\Gamma$. Hence $G^{\prime} \simeq W / \Gamma$. Now we take a 1-dimensional complex subspace $W^{\prime}$ of $C^{n}$ such that $C^{n}=W+W^{\prime}$ (direct sum). Then $G=C^{n} / \Gamma=\left(W+W^{\prime}\right) / \Gamma \simeq W / \Gamma \times W^{\prime} \simeq G^{\prime} \times C$.

(2) Assume that $G / G^{\prime} \simeq C^{*}$.

In this case $W+\Gamma / W$ must be an infinite cyclic group, say, $W+\Gamma / W=Z \bar{a}$, where $\bar{a}$ means the coset of $W+\Gamma / W$ containing the element $a \in \Gamma$. Clearly $a \notin W$. Now put $\Gamma_{1}=\Gamma \cap W$. We assert that $\Gamma=\Gamma_{1}+Z a$ (direct sum). In fact, take an element $b \in \Gamma$, then $\psi(b)=n \psi(a)$ for some $n \in Z$, where $\psi$ is the natural homomorphism of $C^{n}$ onto $C^{n} / W$. Then $b-n a \in \Gamma \cap W=\Gamma_{1}$, and so $b \in \Gamma_{1}+Z a$. We see easily that $\Gamma_{1} \cap Z a=\{0\}$, since $a \notin W$. Since $C^{n}=W+C a$, we see that $G=C^{n} / \Gamma=W / \Gamma_{1}+C a / Z a \simeq G^{\prime} \times C^{*}$. Thus Lemma 3.1 is proved.

THEOREM 3.2. Let $G$ be a connected complex abelian Lie group. Then $G$ is isomorphic to the direct product $G^{0} \times C^{m} \times C^{* n}(m, n \geqq 0)$.

Proof. Since $G / G^{0}$ is a Stein group, $G / G^{0} \simeq C^{m} \times C^{* n}$ by Proposition 4 [4]. We prove the theorem by induction on $m+n$. Suppose that $m \geqq 1$. Take a connected complex subgroup $G_{1}$ of $G$ such that $\phi\left(G_{1} / G^{0}\right)=C^{m-1} \times C^{* n}$, where $\phi$ denotes the isomorphism of $G / G^{0}$ onto $C^{m} \times C^{* n}$. Then $G / G_{1} \simeq C$. Therefore, by Lemma 3.1, we have $G \simeq G_{1} \times C$. Now by the induction assumption $G_{1} \cong G^{0} \times C^{m-1} \times C^{* n}$, where we have used the fact that $\left(G_{1}\right)^{0}=G^{0}$. Hence we obtain $G \simeq G^{0} \times C^{m} \times C^{* n}$. In the case $m=0, n \geqq 1$ we can see in the same argument $G \simeq G^{0} \times C^{m} \times C^{* n}$ by using Lemma 3.1 for the case $G / G_{1} \simeq C^{*}$. Thus Theorem 3.2 is proved. 
COROLlaRy 3.3. Let $G$ be a connected complex abelian Lie group. Then $G$ is holomorphically convex if and only if $G \simeq T \times C^{m} \times C^{* n}(m, n \geqq 0)$, where $T$ is a complex torus.

Proof. By Theorem 2 of [5] $G$ is holomorphically convex if and only if $G^{0}$ is compact, i.e. $G^{0}=T$ is a complex torus. Hence we can apply Theorem 3.2.

Proposition 3.4. Let $G_{1}^{0}, G_{2}^{0}$ be (H.C)-groups and let $S_{1}, S_{2}$ be Stein groups. Put $G_{i}=G_{i}^{0} \times S_{i}$ for $i=1,2$, . Then, $G_{1} \simeq G_{2}$ if and only if $G_{1}^{0} \simeq G_{2}^{0}$ and $S_{1} \simeq S_{2}$.

Proof. Suppose $\operatorname{dim} G_{1}^{0} \geqq \operatorname{dim} G_{2}^{0}$. If $\phi$ is an isomorphism of $G_{1}$ onto $G_{2}$, put $\phi\left(G_{1}^{0}\right)=G_{1}^{\prime}$. Then $G_{1}^{\prime} \subset G_{2}^{0}$ since $\left(G_{i}\right)^{0}=G_{i}^{0}(i=1,2)$. Hence $G_{1}^{\prime}=G_{2}^{0}$. Then $\phi$ induces an isomorphism of $G_{1} / G_{1}^{0}$ onto $G_{2} / G_{2}^{0}$. Thus Proposition 3.4 is proved.

From Proposition 3.4 we obtain the following

Corollary 3.5. Let $G_{1}$ and $G_{2}$ be connected complex abelian groups. Let $G_{i}=G_{i}^{0} \times C^{m_{i}} \times C^{* n_{i}}(i=1,2)$ be the decomposition as in Theorem 3.2. Then $G_{1} \simeq G_{2}$ if and only if $G_{1}^{0} \simeq G_{2}^{0}$ and $m_{1}=m_{2}, n_{1}=n_{2}$.

REMARK 3.6. By Corollary 3.5 we see that in order to classify connected complex abelian Lie groups it is sufficient to classify all (H.C)-groups.

\section{Characterization of (H.C)-groups.}

Lemma 4.1. Let $V \in \mathfrak{M}^{*}(n, m ; C)$ and $G=C^{n} / \Gamma(V)$. Suppose there exists an element $V^{\prime \prime} \in \mathfrak{M}^{*}(n-1, m ; C)$ such that $G \simeq C^{n} / \Gamma\left(V^{\prime}\right)$, where

$$
V^{\prime}=\left(\begin{array}{l}
V^{\prime \prime} \\
0
\end{array}\right) \in \mathfrak{M}(n, m ; C) .
$$

Then, there exists a nonzero vector $x \in Q^{n+m}$ such that

$$
\left({ }^{t} V, E_{m}\right) x=0 .
$$

Proof. Since $C^{n} / \Gamma(V) \simeq C^{n} / \Gamma\left(V^{\prime}\right)$, there exists, by Lemmas 2.3 and 2.4 a matrix $M \in \mathrm{GL}(n+m, Z)$ such that

$$
\left(A+V^{\prime} B\right) V=C+V^{\prime} D
$$

where we have put

$$
M=\left(\begin{array}{ll}
A & C \\
B & D
\end{array}\right)
$$

with $A \in \mathfrak{M}(n, C)$. From (4.2) we have

$$
A V-C=V^{\prime}(D-B V)=\left(\begin{array}{c}
V^{\prime \prime}(D-B V) \\
0
\end{array}\right)
$$


Putting $V^{m}=V^{\prime \prime}(D-B V)$, we have

$$
A V-C=\left(\begin{array}{c}
V^{\prime \prime} \\
0
\end{array}\right)
$$

Now we put

$$
A=\left(\begin{array}{c}
A^{\prime} \\
a
\end{array}\right)
$$

with $A^{\prime} \in \mathfrak{M}(n-1, n ; Z), a \in Z^{n}$ and

$$
C=\left(\begin{array}{c}
C^{\prime} \\
-c
\end{array}\right)
$$

with $C^{\prime} \in \mathfrak{M}(n-1, m ; Z), c \in Z^{m}$. Then from (4.3) we have

$$
{ }^{t} a V+{ }^{t} c=0 \text {. }
$$

Putting

$$
\left(\begin{array}{l}
a \\
c
\end{array}\right)=x,
$$

we obtain $\left({ }^{t} V, E_{m}\right) x=0$. Thus Lemma 4.1 is proved.

LEMma 4.2. Let $G=C^{n} / \Gamma(V)$ with $V \in \mathfrak{M}^{*}(n, m ; C)$. Suppose there exists a nonzero vector $x \in Q^{n+m}$ such that (4.1)holds. Then there exists $V^{\prime \prime} \in \mathfrak{M}^{*}(n-1, m ; C)$ such that $G \simeq C^{n} / \Gamma\left(V^{\prime}\right)$ with

$$
V^{\prime}=\left(\begin{array}{c}
V^{\prime \prime} \\
0
\end{array}\right) \in \mathfrak{M}^{*}(n, m ; C),
$$

where $0=(0, \cdots, 0) \in \mathfrak{M}(1, m ; C)$.

Proof. By (4.1) we can suppose that there exist $0 \neq a \in Z^{n}, c \in Z^{m}$ such that

$$
a V=c \text {. }
$$

Moreover, by changing indices $\{1,2, \cdots, n\}$ and by multiplying rational numbers if necessary, we can suppose that $a=\left(a_{1}, \cdots, a_{n}\right), a_{1} \neq 0$ and $\left(a_{1}, a_{2}, \cdots, a_{n+m}\right)=1$ (coprime), where we have put $c=\left(a_{n+1}, \cdots, a_{n+m}\right)$. Put

$$
\begin{array}{ll}
p_{1}=\left(a_{1}, \cdots, a_{n+m-1}\right), a_{k}=p_{1} a_{k}^{1} \quad(k=1,2, \cdots, n+m-1), \\
p_{2}=\left(a_{1}^{1}, a_{2}^{1}, \cdots, a_{n+m-2}^{1}\right), a_{k}^{1}=p_{2} a_{k}^{2} & (k=1,2, \cdots, n+m-2), \\
p_{3}=\left(a_{1}^{2}, a_{2}^{2}, \cdots, a_{n+m-3}^{2}\right), a_{k}^{2}=p_{3} a_{k}^{3} & (k=1,2, \cdots, n+m-3),
\end{array}
$$

and so on. Then we have $\left(p_{1}, a_{n+m}\right)=1,\left(p_{2}, a_{n+m-1}^{1}\right)=1,\left(p_{3}, a_{n+m-2}^{2}\right)=1$ and so on. Hence there exist $s_{i}, t_{i} \in Z$ such that

$$
t_{i+1} p_{i+1}-s_{i+1} a_{n+m-i}^{i}=1
$$


for $i=0,1, \cdots, n+m-1$, where we put $a_{n+m}^{0}=a_{n+m}$. In this process, we take $s_{i+1}=\mp 1, \quad t_{i+1}=0$ if $a_{n+m-i}^{i}= \pm 1$ holds. Next, put $b_{i j}=s_{i} a_{j}^{l}$ for $j=1,2, \cdots, n+m-i ; i=1,2, \cdots, n+m-1$. Let $M^{\prime}$ be the matrix obtained by replacing $n$ by $n+m$ in (2.4). Then by Lemma $2.6 M^{\prime} \in \mathrm{GL}(n+m, Z)$. Put

$$
M^{\prime}=\left(\begin{array}{ll}
A^{\prime} & C^{\prime} \\
B & D
\end{array}\right)
$$

with $A^{\prime} \in \mathfrak{M}(n, Z)$. Then we have

$$
B=\left[\begin{array}{c}
r_{m} a \\
r_{m-1} a \\
\vdots \\
r_{1} a
\end{array}\right], r_{i}=\frac{s_{i}}{p_{i}}
$$

for $i=1,2, \cdots, m$. Put $C(k)=\left(a_{n+1}, \cdots, a_{n+m-k}, 0, \cdots, 0\right) \in Z^{m}$ for $k=1,2, \cdots, m-1$. Then we have

$$
D=\left[\begin{array}{l}
t_{m} e_{1} \\
t_{m-1} e_{2}+r_{m-1} C(m-1) \\
t_{m-2} e_{3}+r_{m-2} C(m-2) \\
\vdots \\
t_{1} e_{m}+r_{1} C(1)
\end{array}\right]
$$

where $e_{i}(i=1, \cdots, m)$ are unit vectors in $C^{m}$.

We assert that $\operatorname{det}(B V-D) \neq 0$. In fact, by using (4.4), (4.6) and (4.7) we have

$$
\begin{aligned}
& B V-D=\left[\begin{array}{c}
r_{m} a \\
\vdots \\
r_{1} a
\end{array}\right] V-D=\left[\begin{array}{c}
r_{m} C \\
\vdots \\
r_{1} C
\end{array}\right]-\left[\begin{array}{l}
t_{m} e_{1} \\
t_{m-1} e_{2}+r_{m-1} C(m-1) \\
t_{1} e_{m}+r_{1} C(1)
\end{array}\right]
\end{aligned}
$$

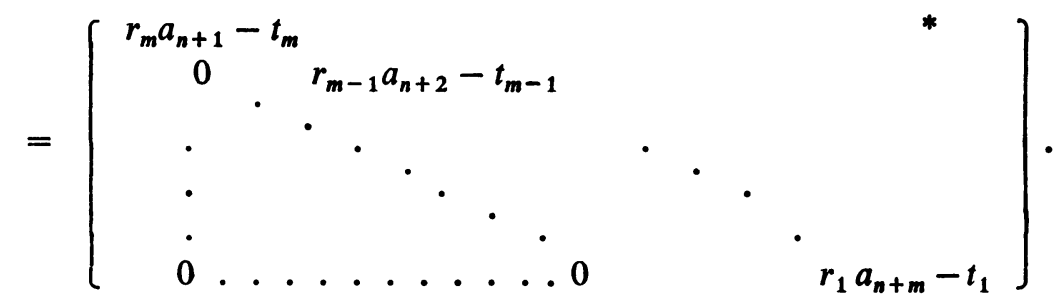

Hence, if $\operatorname{det}(B V-D)=0$, there exists an index $i(1 \leqq i \leqq m)$ such that $r_{i} a_{n+m-i+1}-t_{i}=0$. By (4.6) we have

$$
s_{i} a_{n+m-i+1}-p_{i} t_{i}=0 \text {. }
$$


On the other hand, we see that $a_{n+m-i+1}=p a_{m+n-i+1}^{i-1}$, where we have put $p=p_{1} p_{2} \cdots p_{i-1}$. Hence (4.8) is written as follows

$$
s_{i} p a_{m+n-i+1}^{i-1}-p_{i} t_{i}=0
$$

By (4.5) we have

$$
-s_{i} a_{m+n-i+1}^{i-1}+p_{i} t_{i}=1 .
$$

Adding (4.9) to (4.10) we obtain

$$
s_{i} a_{m+n-i+1}^{i-1}(p-1)=1 .
$$

Since all numbers in the left-hand side of (4.11) are integers, we see that

$$
a_{m+n-i+1}^{i-1}= \pm 1 \text {. }
$$

Then, by the process of finding $s_{i}, t_{i}$, we have $t_{i}=0$ and $s_{i}=\mp 1$. Then by (4.9) we obtain $( \pm 1) p( \pm 1)=0$, which is a contradiction. Thus we have proved our assertion $\operatorname{det}(B V-D) \neq 0$.

Now we denote by $M$ the matrix obtained from $M^{\prime}$ by exchanging the first row and the $n$th row. Put

$$
M=\left(\begin{array}{ll}
A & C \\
B & D
\end{array}\right)
$$

with $A \in \mathfrak{M}(n, Z)$. Then the $n$th row of $C-A V$ is $c-a V=0$. Hence, if we define $V^{\prime}$ by $V^{\prime}=(C-A V)(B V-D)^{-1}$, we see that the $n$th row of $V^{\prime}$ is zero. Moreover, by the definition of $V^{\prime}$, we have $V^{\prime}(B V-D)=C-A V$, and so $\left(A+V^{\prime} B\right) V=C+V^{\prime} D$. Hence, by Lemmas $2.2-2.4$, we see that $V^{\prime} \in \mathfrak{M}^{*}(n, m ; C)$ and that $G=C^{n} / \Gamma(V) \simeq C^{n} / \Gamma\left(V^{\prime}\right)$. Thus Lemma 4.2 is proved.

Proposition 4.3. Let $G=C^{n} / \Gamma(V)$ with $V \in \mathfrak{M}^{*}(n, m ; C)$. Then, $G$ contains a closed complex subgroup $G_{1}$ such that $G \simeq G_{1} \times C^{*}$ if and only if there exists a nonzero vector $x \in Q^{n+m}$ such that (4.1) holds.

Proof. If $G \simeq G_{1} \times C^{*}$, by Corollary 3.5 , we see that $G_{1} \simeq C^{n-1} / \Gamma\left(V_{1}\right)$ for some $V_{1} \in \mathfrak{M}^{*}(n-1, m ; C)$. Putting

$$
V^{\prime}=\left(\begin{array}{c}
V_{1} \\
0
\end{array}\right) \in \mathfrak{M}^{*}(n, m ; C),
$$

$G \simeq C^{n} / \Gamma\left(V^{\prime}\right)$. By Lemma 4.1 there exists a nonzero vector $x \in Q^{n+m}$ such that (4.1) holds. The converse is also true by Lemma 4.2. Thus Proposition 4.3 is proved.

We can now characterize (H.C)-groups as follows (cf. [2]).

THEOREM 4.4. Let $G$ be an (H.C)-group of dimension n. Then $G$ is isomorphic to $C^{n} / \Gamma(V)$ for some $V \in \mathfrak{M}^{*}(n, m ; C)$ satisfying the following condition:

$$
x \in Q^{n+m},\left({ }^{t} V, E_{m}\right) x=0 \text { imply } x=0 .
$$


Conversely any such group $C^{n} / \Gamma(V)$ satisfying (4.12) is an (H.C)-group.

Proof. If $G$ is an (H.C)-group, then $G$ is a connected complex abelian Lie group (§2). Hence $G \simeq C^{n} / \Gamma$ for some discrete subgroup of $\boldsymbol{C}^{n}$. Consider the complex subspace $U$ of $C^{n}$ spanned by $\Gamma$. We assert that $U=C^{n}$. If not, there exists a complex subspace $W \neq\{0\}$ of $C^{n}$ such that $C^{n}=U+W$ (direct sum). Then $G \simeq C^{n} / \Gamma \simeq U / \Gamma \times W$, which is a contradiction. Since $U=C^{n}$, we can find a system of generators $s_{1}, \cdots, s_{n+m}$ of $\Gamma$ such that $s_{1}, \cdots, s_{n}$ are linearly independent over $C$. Take an automorphism $\phi$ of $C^{n}$ such that $\phi\left(s_{i}\right)=e_{i}$ $(i=1,2, \cdots, n)$ and put $\phi\left(s_{n+j}\right)=v_{j}(j=1,2, \cdots, m)$. Then it is clear that $G \simeq \boldsymbol{C}^{n} / \Gamma(V)$ for $V=\left(v_{1}, \cdots, v_{m}\right) \in \mathfrak{M}^{*}(n, m ; C)$. Now, by Proposition 4.3 the matrix $V$ satisfies (4.12).

Conversely, suppose $G=C^{n} / \Gamma(V)$ satisfies (4.12). If $G$ is not an (H.C)-group, by Theorem 3.2, $G \simeq G^{0} \times C^{l} \times C^{* m}$ for some $l, m$ with $l+m>0$. We shall see that $l=0$. In fact, if $l>0$, put $G^{0}=C^{n_{0}} / \Gamma\left(V_{0}\right)$ for $n_{0}=n-(l+m)$, $V_{0} \in \mathfrak{M}^{*}\left(n_{0}, m_{0} ; C\right)$. Then $G^{0} \times C^{l} \times C^{* m} \simeq C^{n} / \Gamma$, where $\Gamma$ contains only $n-l$ vectors of $\boldsymbol{C}^{n}$ linearly independent over $\boldsymbol{C}$, which is a contradiction. Since $l=0$, $G \cong G^{0} \times C^{* m}(m>0)$. Then by Proposition 4.3 , there exists a nonzero vector $x \in Q^{n+m}$ satisfying (4.1), which is again a contradiction. Thus Theorem 4.4 is proved.

5. Classification of (H.C)-groups of dimension $\boldsymbol{n}$ and of rank $n+1$.

Definition 5.1. Let $\left(\boldsymbol{C}^{n}-\boldsymbol{R}^{n}\right)^{\prime}$ be the set of all $v={ }^{t}\left(\alpha_{1}, \cdots, \alpha_{n}\right) \in \boldsymbol{C}^{n}-\boldsymbol{R}^{n}$ such that $1, \alpha_{1}, \cdots, \alpha_{n}$ are linearly independent over $\boldsymbol{Q}$. For $M=\left(a_{i j}\right) \in \operatorname{GL}(n+1, Z)$ and $v={ }^{t}\left(\alpha_{1}, \cdots, \alpha_{n}\right) \in\left(C^{n}-R^{n}\right)^{\prime}$ we define $v^{\prime}=\tilde{M}(v)={ }^{t}\left(\alpha_{1}^{\prime}, \cdots, \alpha_{n}^{\prime}\right) \in C^{n}$ as follows:

$$
\alpha_{i}^{\prime}=\left(a_{i, n+1}-\sum_{j=1}^{n} a_{i j} \alpha_{j}\right) /\left(\sum_{j=1}^{n} a_{n+1, j} \alpha_{j}-a_{n+1, n+1}\right)
$$

for $i=1,2, \cdots, n$. We note that the denominator of (5.1) does not vanish since $1, \alpha_{1}, \cdots, \alpha_{n}$ are linearly independent over $\boldsymbol{Q}$.

Lemma 5.2. Let $v \in C^{n}-R^{n}$. Then, $C^{n} / \Gamma(v)$ is an (H.C)-group if and only if $v \in\left(\boldsymbol{C}^{n}-\boldsymbol{R}^{n}\right)^{\prime}$.

In fact, we see readily that the condition (4.12) for $m=1$ means that $1, \alpha_{1}, \cdots, \alpha_{n}$ are linearly independent over $\boldsymbol{Q}$.

Lemma 5.3. Let $v \in\left(C^{n}-R^{n}\right)^{\prime}$ and $M \in \mathrm{GL}(n+1, Z)$. Then $\tilde{M}(v) \in\left(C^{n}-R^{n}\right)^{\prime}$.

Proof. Put $v^{\prime}=\tilde{M}(v)$. By a simple calculation we see that the equalities (5.1) mean $\left(A+v^{\prime} B\right) v=C+d v^{\prime}$ for

$$
M=\left(\begin{array}{ll}
A & C \\
B & d
\end{array}\right),
$$


where $A \in M(n, Z)$. Then by Lemma $2.4, C^{n} / \Gamma(v) \simeq C^{n} / \Gamma\left(v^{\prime}\right)$ holds. Hence, by Lemma 5.2, $C^{n} / \Gamma\left(v^{\prime}\right)$ is an (H.C)-group and so $v^{\prime} \in\left(C^{n}-R^{n}\right)^{\prime}$.

LEMMA 5.4. Let $v, v^{\prime} \in\left(\boldsymbol{C}^{n}-\boldsymbol{R}^{n}\right)^{\prime}$. Then, $\boldsymbol{C}^{n} / \Gamma(v) \simeq \boldsymbol{C}^{n} / \Gamma\left(v^{\prime}\right)$ if and only if there exists an element $M \in \mathrm{GL}(n+1, Z)$ such that $v^{\prime}=\tilde{M}(v)$.

In fact, the condition (2.1) for $m=1$ is equivalent to (5.1) if $1, \alpha_{1}, \cdots, \alpha_{n}$ are linearly independent over $\boldsymbol{Q}$. Thus Lemma 5.4 is proved.

Since it is easily seen that $\left[M M^{\prime}\right]^{\sim}=\tilde{M} \circ \tilde{M}^{\prime}$ for $M, M^{\prime} \in \mathrm{GL}(n+1, Z)$ we have the following

LEMMA 5.5. The group $\mathrm{GL}(n+1, Z)$ operates on $\left(C^{n}-R^{n}\right)^{\prime}$ by the action $(M, v) \rightarrow \tilde{M}(v)$.

Definition 5.6. We denote by $\boldsymbol{A}(n)$ the set of all complex abelian Lie groups $\boldsymbol{C}^{n} / \Gamma(v)$ with $v \in \boldsymbol{C}^{n}-\boldsymbol{R}^{n}$ (two isomorphic ones being of course identified).

We can now classify $A(n)$ as follows.

THEOREM 5.7. We denote by $\boldsymbol{A}^{\circ}(n)\left(\boldsymbol{A}^{\prime}(n)\right.$ resp.) the subset of all (H.C)-groups (non (H.C)-groups resp.) in $A(n)$. Then $A(n)$ can be classified as follows:

$$
\begin{aligned}
& A(n)=A^{0}(n) \cup A^{\prime}(n)(\text { disjoint }), \\
& A^{\prime}(n)=A(n-1) \times\left\{C^{*}\right\}, \\
& A^{0}(n)=\left(C^{n}-R^{n}\right)^{\prime} / \mathrm{GL}(n+1, Z) .
\end{aligned}
$$

In fact, if $G \in A^{\prime}(n)$, then by Theorem $3.2 G \simeq G^{0} \times C^{l} \times C^{* m}$. However, we see $l=0$ by the same argument as in the proof of Theorem 4.4. Hence $G \simeq G_{1} \times C^{*}$ for $G_{1}=G^{0} \times C^{* m-1}$. The group $G_{1}$ is easily seen to be an element of $A(n-1)$. On the other hand, it is seen by Lemmas 5.2-5.5 that there exists a natural one-one correspondence between $A^{0}(n)$ and the quotient space of $\left(C^{n}-R^{n}\right)^{\prime}$ by the action of $\operatorname{GL}(n+1, Z)$. Thus Theorem 5.7 is proved.

\section{Nonexistence of compact complex submanifolds.}

THEOREM 6.1. Let $G=C^{n} / \Gamma(v)$ be an element of $A^{0}(n)$. Then $G$ contains no compact complex submanifold of positive dimension.

Proof. Let $U$ be the real subspace of $C^{n}$ spanned by $\Gamma(v)$ over $R$, and let $W$ be the real subspace of $C^{n}$ such that $C^{n}=U+W$ (direct sum). Then $G$ is isomorphic to $K \times W=K \times R^{n-1}$ as a real Lie group, $K$ being the maximal compact subgroup of $G$. Let $f_{i}(i=1,2, \cdots, n-1)$ be the real-valued function on $G$ defined by $f_{i}\left(k, x_{1}, \cdots, x_{n-1}\right)=x_{i}$ for $k \in K, x_{i} \in R$. Then $f_{i}$ is a plurisubharmonic function on $G$, since $\partial^{2} f / \partial Z_{\mu} \partial Z_{v}=0$ for $\mu, v=1, \cdots, n,\left\{Z_{1}, \cdots, Z_{n}\right\}$ being the complex coordinates on $C^{n}$. Now suppose that there exists a compact complex submanifold $X$ in $G$. Then the plurisubharmonic function $f_{i} \mid X$ on $X$ must be a constant $a_{i}$ (cf. e.g. [3]). Put $a=\left(a_{1}, \cdots, a_{n-1}\right)$. Then $X$ is contained in $K \times\{a\}$. 
Hence we can suppose that $X$ is contained in $K=K \times\{0\}$. Since $X$ is a complex submanifold of $G$, the tangent space $T_{e}(x)$ of $X$ at $e$ is contained in the maximum complex subspace $\mathfrak{f}_{0}$ of the tangent space $T_{e}(K)$ of $K$ at $e$. Since $\operatorname{dim}_{c} \mathfrak{f}_{0}=1$, $\operatorname{dim}_{C} X \leqq 1$. Suppose $\operatorname{dim}_{C} X=1$. Now, for $x \in K$, let $D_{x}$ be the maximum complex subspace of the tangent space $T_{x}(K)$ of $K$ at $x$. It is clear that the assignment $x \rightarrow D_{x}$ is a real 2-dimensional involutive distribution $D$ on $K$ in the sense of Chevalley [1]. Then $X$ is an integral manifold of $D$. Since $X$ is compact, $X$ is a maximal integral manifold of $D$, containing the unit element $e$. On the other hand $K_{0}$ is a maximal integral manifold of $D$ containing $e$, where $K_{0}$ is the subgroup of $G$ corresponding to the Lie subalgebra $\mathfrak{f}_{0}$. Hence $X$ and $K_{0}$ must coincide by a theorem of [1]. Hence $K_{0}$ must be compact. Then by Theorem 2 [5] $G$ is holomorphically convex and hence $G$ is not an (H.C)-group, which is a contradiction. Thus Theorem 6.1 is proved.

LeMmA 6.2. Let $K$ be a Lie group. Let $D=\left\{D_{x}\right\}$ be an involutive distribution on $K$ invariant under left translations on $K$. Let $X$ be a closed integral manifold of $D$ containing the unit element $e$, i.e. $T_{x}(X) \subset D_{x}$ for any $x \in X$. Then there exist a neighborhood $U$ of $e$ in $K$ and an integral manifold $X^{\prime}$ of $D$ in $U$ such that $X^{\prime} \supset X \cap U$ and that $\operatorname{dim} X^{\prime}=\operatorname{dim} D$.

Proof. Let $K_{0}$ be the maximal integral manifold of $D$ containing $e$ (dim $K_{0}$ $=\operatorname{dim} D$ ). Then $K_{0}$ is a subgroup of $K$. If $\operatorname{dim} X=\operatorname{dim} D$, the lemma is clearly true. Suppose $\operatorname{dim} X<\operatorname{dim} D=\operatorname{dim} T_{e}\left(K_{0}\right)$. Take an element $Y_{1} \in T_{e}\left(K_{0}\right)-T_{e}(X)$. Then there exists a neighborhood $U_{1}$ of $e$ such that $L_{a}^{\prime} Y_{1} \notin T_{a}(X)$ for $a \in U_{1} \cap X$, where $L_{a}^{\prime}$ denotes the differential of the left translation $L_{a}$ corresponding to $a \in K$. Clearly $L_{a}^{\prime} Y_{1} \in D_{a}$ for any $a \in K$. We shall denote by exp $t Y_{1}$ the one-parameter subgroup of $K$ whose tangent vector at $e$ is $Y_{1}$. Now we put

$$
X_{1}=\left\{\left(\exp t Y_{1}\right) x\left|x \in X \cap U_{1},\right| t \mid<\varepsilon_{1}\right\} .
$$

Then $X_{1}$ is an integral manifold of $D$ for sufficiently small $\varepsilon_{1}>0$. If $\operatorname{dim} X_{1}<\operatorname{dim} D$, take again an element $Y_{2} \in T_{e}\left(K_{0}\right)-T_{e}\left(X_{1}\right)$. Then there exists a neighborhood $U_{2}$ of $e$ such that $L_{a}^{\prime} Y_{2} \notin T_{a}(X)$ for $a \in U_{2} \cap X_{1}$. Put

$$
X_{2}=\left\{\left(\exp t Y_{2}\right) x\left|x \in W_{1} \cap U_{2},\right| t \mid<\varepsilon_{2}\right\} .
$$

Then $X_{2}$ is an integral manifold of $D$ for sufficiently small $\varepsilon_{2}>0$. If $\operatorname{dim} X_{2}<\operatorname{dim} D$ take an element $Y_{3} \in T_{e}\left(K_{0}\right)-T_{e}\left(X_{2}\right)$ and so on. We can finally find a neighborhood $U$ and an integral manifold $X^{\prime}$ satisfying the required properties. Thus Lemma 6.2 is proved.

COROLlaRY 6.3. Notations being as in Lemma 6.2, and $K_{0}$ being the maximal integral manifold of $D$ containing $e$, we have $X \subset K_{0}$, if $X$ is connected.

Proof. Take a point $p \in X$, then $p^{-1} X$ is an integral manifold of $D$ containing $e$. Therefore, by Lemma 6.2, we can find a neighborhood $U_{p}$ of $e$ and an integral 
manifold $X_{p}$ of $D$ in $U_{p}$ (depending on $p$ ) such that $U_{p} \cap p^{-1} X \subset X_{p} \subset K_{0}$. Put $p X_{p}=K_{p}$. Then $K_{p}$ is an integral manifold of $D$ containing $p$ and $\operatorname{dim} K_{p}$ $=\operatorname{dim} K_{0}$. Now put $X_{0}=\left\{p \in X \mid K_{p} \subset K_{0}\right\}$. It is clear that $e \in X_{0}$ and that $X_{0}$ is open in $X$. We shall prove that $X_{0}$ is closed in $X$. Take $p_{0} \in X_{0} \cap X$. Since $K_{p_{0}} \cap X$ is open in $X$, there is a point $p_{1} \in K_{p_{0}} \cap X_{0}$. Then, since $K_{p_{0}}$ and $K_{p_{1}}$ are both integral manifolds of $D$ containing $p_{1}$ and since $\operatorname{dim} K_{p_{0}}=\operatorname{dim} K_{p_{1}}=\operatorname{dim} D$, there exists a neighborhood $U$ of $p_{1}$ such that $K_{p_{1}} \cap U=K_{p_{0}} \cap U$. On the other hand, since $p_{1} \in X_{0}, K_{p_{1}} \subset K_{0}$ holds. Hence $K_{p_{0}} \cap U \subset K_{0}$ and so $K_{p_{0}} \subset K_{0}$ since $K_{0}$ is a maximal integral manifold of $D$. Therefore $p_{0} \in X_{0}$, whence $X_{0}$ is closed in $X$. Since $X$ is connected $X=X_{0}$ holds, which implies $X \subset K_{0}$. Thus Corollary 6.3 is proved.

THEOREM 6.4. Let $G$ be a connected complex abelian Lie group. If $G$ contains no complex torus of positive dimension, then $G$ contains no compact complex submanifold of positive dimension.

Proof. Suppose that there exists a connected compact complex submanifold $X$ in $G$ containing $e$. Let $K, K_{0}$ be the same subgroups of $G$ and $D=\left\{D_{x}\right\}$ be the same distribution on $K$ as in the proof of Theorem 6.1, which shows that $X \subset K$ and that $X$ is an integral manifold of $D$. Then by Corollary 6.3 we have $X \subset K_{0}$. Let $K^{\prime}$ be the maximal compact subgroup of $K_{0}$ and $\mathfrak{f}^{\prime}$ be the Lie algebra of $K^{\prime}$. Put $\mathfrak{f}_{1}=\mathfrak{f}^{\prime} \cap \sqrt{ }(-1) \mathfrak{f}^{\prime}$ and take the subgroup $K_{1}$ of $K$ corresponding to $\mathfrak{f}_{1}$. Then by the same argument as above we see that $X \subset K_{1}$. We consider again the maximal compact subgroup $K^{\prime \prime}$ of $K_{1}$ and let $\mathfrak{l}^{\prime \prime}$ be the Lie algebra of $K^{\prime \prime}$. Put $\mathfrak{f}_{2}=\mathfrak{f}^{\prime \prime} \cap \sqrt{ }(-1) \mathfrak{f}^{\prime \prime}$ and take the subgroup $K_{2}$ corresponding to $\mathfrak{f}_{2}$, and so on. Thus we have a sequence of complex subgroups $K_{1} K_{2} \cdots$ such that $K_{i} \supset X$ for $i=1,2, \cdots$. Since $G$ does not contain any complex torus of positive dimension, we see that $K_{i} \neq K_{i+1}$ if $\operatorname{dim} K_{i}>0$. Hence we have $X=\{e\}$. Thus Theorem 6.4 is proved.

REMARK 6.5. We see that the group $G$ constructed in Theorem 3 [5] contains no compact complex submanifold of positive dimension.

REMARK 6.6. Using the fact that the set of all singular points of a complex analytic set is also an analytic set, we can prove that the group in Theorem 6.4 contains no compact complex analytic set of positive dimension.

7. Nonsingular $n \times m$ matrices with $n>m$.

Definition 7.1. We shall call a matrix $V \in \mathfrak{M}(n, m ; C)(n>m)$ nonsingular if $\operatorname{det}(A+V B) \neq 0$ for any

$$
M=\left(\begin{array}{ll}
A & C \\
B & D
\end{array}\right) \in \mathrm{GL}(n+m, Z)
$$

with $A \in \mathfrak{M}(n, Z)$. Otherwise $V$ will be called singular. 
LEMMA 7.2. A matrix $V \in \mathfrak{M}(n, m ; C)(n>m)$ is nonsingular if and only if $\operatorname{det}(D-B V) \neq 0$ for all

$$
M=\left(\begin{array}{ll}
A & C \\
B & D
\end{array}\right) \in \mathrm{GL}(n+m, Z)
$$

with $A \in \mathfrak{M}(n, Z)$.

Proof. Assume $V$ is nonsingular. Take $M \in \mathrm{GL}(n+m, Z)$ and put

$$
M=\left(\begin{array}{ll}
A & C \\
B & D
\end{array}\right), \quad M^{-1}=\left(\begin{array}{ll}
A^{\prime} & C^{\prime} \\
B^{\prime} & D^{\prime}
\end{array}\right)
$$

with $A, A^{\prime} \in \mathfrak{M}(n, Z)$. Since $\operatorname{det}\left(A^{\prime}+V B^{\prime}\right) \neq 0$, we can define $V^{\prime} \in \mathfrak{M}(n, m ; C)$ by

$$
\left(A^{\prime}+V B^{\prime}\right) V^{\prime}=C^{\prime}+V D^{\prime} \text {. }
$$

The equality (7.1) is equivalent, by Lemma 2.3 , to the equality $\left(A+V^{\prime} B\right) V=C+V^{\prime} D$, which implies $A V-C=V^{\prime}(D-B V)$, which is rewritten as

or

$$
\left(\begin{array}{ll}
A & C \\
B & D
\end{array}\right)\left(\begin{array}{c}
V \\
-E_{m}
\end{array}\right)=\left(\begin{array}{c}
V^{\prime} \\
-E_{m}
\end{array}\right)(D-B V)
$$

$$
{ }^{t}(D-B V)\left({ }^{t} V^{\prime},-E_{m}\right)=\left({ }^{t} V,-E_{m}\right)^{t} M,
$$

which is equivalent to

$$
{ }^{t}(D-B V)\left({ }^{t} V^{\prime},-E_{m}\right)^{t} M^{-1}=\left({ }^{t} V,-E_{m}\right) .
$$

Now, put ${ }^{t}\left(V^{\prime},-E_{m}\right)^{t} M^{-1}=\left(u_{1}, \cdots, u_{n+m}\right)$ with $u_{i} \in C^{m}$ for $i=1,2, \cdots, n+m$. Then, we have ${ }^{t}(D-B V) u_{n+i}=-e_{i} \quad(i=1, \cdots, m)$, which shows that $\operatorname{det}(D-B V) \neq 0$. Conversely if $\operatorname{det}(D-B V) \neq 0$ for any

$$
M=\left(\begin{array}{ll}
A & C \\
B & D
\end{array}\right) \in \mathrm{GL}(n+m, Z)
$$

we can define $V^{\prime}$ by the equality

$$
A^{\prime} V-C^{\prime}=V^{\prime}\left(D^{\prime}-B^{\prime} V\right)
$$

which is equivalent to $(A+V B) V^{\prime}=C+V D$. Hence by Lemma $2.2 \operatorname{det}(A+V B)$ $\neq 0$. Thus Lemma 7.2 is proved.

Lemma 7.3. Let $V \in \mathfrak{M}(n, m ; C)(n>m)$ be nonsingular. Take

$$
M=\left(\begin{array}{ll}
A & C \\
B & D
\end{array}\right) \in \mathrm{GL}(n+m, Z)
$$

with $A \in \mathfrak{M}(n, Z)$ We define $V^{\prime} \in \mathfrak{M}(n, m ; C)$ by

$$
(A+V B) V^{\prime}=C+V D .
$$

Then $V^{\prime}$ is also nonsingular. 
Proof. Take $M_{1} \in \mathrm{GL}(n m, Z)$ and put

with $A_{1} \in \mathfrak{M}(n, Z)$. Put

$$
M_{1}=\left(\begin{array}{ll}
A_{1} & C_{1} \\
B_{1} & D_{1}
\end{array}\right)
$$

$$
M_{2}=M_{1} M^{-1}=\left(\begin{array}{ll}
A_{2} & C_{2} \\
B_{2} & D_{2}
\end{array}\right) \text { and } M_{2}^{-1}=\left(\begin{array}{ll}
A_{2}^{\prime} & C_{2}^{\prime} \\
B_{2}^{\prime} & D_{2}^{\prime}
\end{array}\right)
$$

with $A_{2}, A_{2}^{\prime} \in \mathfrak{M}(n, Z)$. We define $V^{\prime \prime} \in \mathfrak{M}(n, m ; C)$ by

$$
\left(A_{2}^{\prime}+V B_{2}^{\prime}\right) V^{\prime \prime}=C_{2}^{\prime}+V D_{2}^{\prime} \text {. }
$$

We shall prove the following

$$
\left(A_{1}+V^{\prime \prime} B_{1}\right) V^{\prime}=C_{1}+V^{\prime \prime} D_{1} .
$$

Put

$$
M^{-1}=\left(\begin{array}{ll}
A^{\prime} & C^{\prime} \\
B^{\prime} & D^{\prime}
\end{array}\right)
$$

with $A^{\prime} \in \mathfrak{M}(n, Z)$. Then we have

$$
\begin{array}{ll}
A_{2}=A_{1} A^{\prime}+C_{1} B^{\prime}, & C_{2}=A_{1} C^{\prime}+C_{1} D^{\prime}, \\
B_{2}=B_{1} A^{\prime}+D_{1} B^{\prime}, & D_{2}=B_{1} C^{\prime}+D_{1} D^{\prime} .
\end{array}
$$

Now from (7.3) and Lemma 2.3 we have

$$
\left(A_{2}+V^{\prime \prime} B_{2}\right) V=C_{2}+V^{\prime \prime} D_{2} .
$$

Inserting (7.5) into (7.6) we have $\left(A_{1} A^{\prime}+C_{1} B^{\prime}\right) V+V^{\prime \prime}\left(B_{1} A^{\prime}+D, B^{\prime}\right) V$ $=A_{1} C^{\prime}+C_{1} D^{\prime}+V^{\prime \prime}\left(B_{1} C^{\prime}+D_{1} D^{\prime}\right)$, and so

(7.7) $A_{1}\left(A^{\prime} V-C^{\prime}\right)+V^{\prime \prime} B_{1}\left(A^{\prime} V-C^{\prime}\right)=C_{1}\left(D^{\prime}-B^{\prime} V\right)+V^{\prime \prime} D_{1}\left(D^{\prime}-B^{\prime} V\right)$.

On the other hand, from (7.2) and Lemma 2.2 we have $\left(A^{\prime}+V^{\prime} B^{\prime}\right) V=C^{\prime}+V^{\prime} D^{\prime}$. Hence we have

$$
A^{\prime} V-C^{\prime}=V^{\prime}\left(D^{\prime}-B^{\prime} V\right) .
$$

Inserting (7.8) into (7.7) we obtain

$$
\left(A_{1}+V^{\prime \prime} B_{1}\right) V^{\prime}\left(D^{\prime}-B^{\prime} V\right)=\left(C_{1}+V^{\prime \prime} D_{1}\right)\left(D^{\prime}-B^{\prime} V\right) \text {. }
$$

Since $V$ is nonsingular $\operatorname{det}\left(D^{\prime}-B^{\prime} V\right) \neq 0$ by Lemma 7.2. Hence (7.9) implies $\left(A_{1}+V^{\prime \prime} B_{1}\right) V^{\prime}=C_{1}+V^{\prime \prime} D_{1}$, whence (7.4) is proved. Then by Lemma 2.3 we see

$$
\left(A_{1}^{\prime}+V^{\prime} B_{1}^{\prime}\right) V^{\prime \prime}=C_{1}^{\prime}+V^{\prime} D_{1}^{\prime} \text {, }
$$

where we have put 


$$
M_{1}^{-1}=\left(\begin{array}{ll}
A_{1}^{\prime} & C_{1}^{\prime} \\
B_{1}^{\prime} & D_{1}^{\prime}
\end{array}\right)
$$

with $A_{1}^{\prime} \in \mathfrak{M}(n, Z)$. Hence, by Lemma $2.2, \quad \operatorname{det}\left(A_{1}^{\prime}+V^{\prime} B_{1}^{\prime}\right) \neq 0$. Since $M_{1} \in \mathrm{GL}(n+m, Z)$ was arbitrary, we have proved that $V^{\prime}$ is nonsingular.

Definition 7.4. We denote by $\mathfrak{M}^{* *}(n, m ; C)$ the set of all $V \in \mathfrak{M}^{*}(n, m ; C)$ such that $V$ is nonsingular. For

$$
M=\left(\begin{array}{ll}
A & C \\
B & D
\end{array}\right) \in \mathrm{GL}(n+m, Z)
$$

with $A \in \mathfrak{M}(n, Z)$ and for $V \in \mathfrak{M}^{* *}(n, m ; C)$ we define $V^{\prime}=M^{*}(V) \in \mathfrak{M}(n, m ; C)$ by

$$
\left(A^{\prime}+V B^{\prime}\right) V^{\prime}=C^{\prime}+V D^{\prime},
$$

where we have put

with $A^{\prime} \in \mathfrak{M}(n, Z)$.

$$
M^{-1}=\left(\begin{array}{ll}
A^{\prime} & C^{\prime} \\
B^{\prime} & D^{\prime}
\end{array}\right)
$$

LEMmA 7.5. For any $V \in \mathfrak{M}^{* *}(n, m ; C)$ and $M \in \mathrm{GL}(n+m, Z)$, we have $M^{*}(V) \in \mathfrak{M}^{* *}(n, m ; C)$. Moreover for any $M, M_{1} \in \mathrm{GL}(n+m, Z)$ we have $\left(M M_{1}\right)^{*}=M^{*} \circ M_{1}^{*}$.

Proof. The first half is the consequence of Lemma 7.3. Next, we put $V^{\prime}=M^{*}(V)$ and $V^{\prime \prime}=M_{1}^{*}\left(V^{\prime}\right)$. Then we have, by Lemma 2.3 , the following two equalities:

$$
\begin{gathered}
\left(A+V^{\prime} B\right) V=C+V^{\prime} D, \\
\left(A_{1}+V^{\prime \prime} B_{1}\right) V^{\prime}=C_{1}+V^{\prime \prime} D_{1} .
\end{gathered}
$$

Multiplying $D-B V$ from the right to (7.11) we get

$$
\left(A_{1}+V^{\prime \prime} B_{1}\right) V^{\prime}(D-B V)=\left(C+V^{\prime \prime} D_{1}\right)(D-B V) \text {. }
$$

From (7.10) we have $A V-C=V^{\prime}(D-B V)$. Inserting this into (7.12) we obtain

$$
\left(\left(A_{1} A+C_{1} B\right)+V^{\prime \prime}\left(B_{1} A+D_{1} B\right)\right) V=A_{1} C+C_{1} D+V^{\prime \prime}\left(B_{1} C+D_{1} D\right),
$$

which means $\left(M_{1} M\right)^{*}(V)=V^{\prime \prime}=M_{1}^{*}\left(M^{*} V\right)$. Thus Lemma 7.5 is proved.

REMARK 7.6. From the above considerations we see that the group $\mathrm{GL}(n+m, Z)$ operates on $\mathfrak{M}^{* *}(n, m ; C)$ by the action $(M, V) \rightarrow M^{*}(V)$. In the case $m=1<n$, this operation coincides with the one considered in Lemma 5.5.

\section{Nonsingular abelian Lie groups of dimension $n$.}

THEOREM 8.1. Let $V \in \mathfrak{M}^{* *}(n, m ; C)(n>m)$. Then $G=C^{n} / \Gamma(V)$ contains no complex torus of positive dimension as a subgroup. 
Proof. Suppose $G$ contains a complex torus $T$ of dimension $t>0$. We shall prove that there exists $M \in \mathrm{GL}(n+m, Z)$ such that $\operatorname{det}(A+V B)=0$, where

$$
M=\left(\begin{array}{ll}
A & C \\
B & D
\end{array}\right)
$$

with $A \in \mathfrak{M}(n, Z)$.

First we denote by $K$ the maximal compact subgroup of $G$ and $\mathfrak{g}(\mathfrak{f}, t$ resp.) the Lie algebra corresponding to $G\left(K, T\right.$ resp.). Put $\mathfrak{f}_{0}=\mathfrak{f} \cap \sqrt{ }(-1) \mathfrak{f}$. Then it is clear that $\mathrm{t} \subset \mathfrak{f}_{0}$. Identifying $\mathfrak{g}$ with $C^{n}$ we see that $\mathrm{t}$ contains $2 t$ vectors $u_{1}, u_{2}, \cdots, u_{2 t} \in \Gamma$ linearly independent over $R$. We can suppose that $u_{1}, \cdots, u_{t}$ span the vector space $t$ over $C$. We denote by $W$ the real vector subspace of $\boldsymbol{C}^{n}$ spanned by $u_{1}, \cdots, u_{t+1}$ over $\boldsymbol{R}$. We can suppose $W \cap \Gamma$ is generated by $u_{1}, \cdots, u_{t+1}$. Put

$$
u_{i}=\sum_{j=1}^{n} a_{i}^{j} e_{j}+\sum_{k=1}^{m} b_{i}^{k} v_{k},
$$

where $a_{i}^{j}, b_{i}^{k} \in \boldsymbol{Z}$ for $i=1,2, \cdots, t+1 ; j=1, \cdots, n ; k=1, \cdots, m$. We shall prove that there exists a matrix $M \in \mathrm{GL}(n+m, Z)$ such that $M=\left(a_{i j}\right)$ satisfies the following equalities

$$
\begin{aligned}
& a_{i j}=a_{j}^{i}, \quad i=1, \cdots, n ; j=1, \cdots, t+1, \\
& a_{i j}=b_{j}^{i-n}, i=n+1, \cdots, n+m ; j=1, \cdots, t+1 .
\end{aligned}
$$

For such an

$$
M=\left(\begin{array}{ll}
A & C \\
B & D
\end{array}\right)
$$

with $A \in \mathfrak{M}(n, Z)$, we have $A+V B=\left(\alpha_{i j}\right)$ with $\alpha_{i j}=a_{j}^{i}+\sum_{k=1}^{n} b_{j}^{k} \alpha_{i}^{(k)}$, where we have put $V=\left(v_{1}, \cdots, v_{m}\right), t_{v_{i}}=\left(\alpha_{1}^{(i)}, \cdots, \alpha_{n}^{(i)}\right)$. Then, we have $A+V B=$ $\left(u_{1}, \cdots, u_{t+1}, A^{\prime}\right)$ with $A^{\prime} \in \mathfrak{M}(n, n-t-1 ; C)$. Since $u_{t+1} \in \mathfrak{t}=\left\{u_{1}, \cdots, u_{t}\right\}_{c}$, we see that $\operatorname{det}(A+V B)=0$. It is now sufficient to prove the existence of $M$ satisfying (8.1).

We denote by $\phi$ the natural projection of $C^{n}$ onto $C^{n} / W$. The map $\phi$ is of course an $\boldsymbol{R}$-linear map. Since $\phi(\Gamma)$ is finitely generated abelian group without torsion, there exists a system of generators $\left\{\phi\left(x_{1}\right), \cdots, \phi\left(x_{s}\right)\right\}$ of $\phi(\Gamma)$ with $x_{1}, \cdots, x_{s} \in \Gamma$ such that every element $a$ of $\phi(\Gamma)$ can be written uniquely as

$$
a=\sum_{i=1}^{s} a_{i} \phi\left(x_{i}\right), \quad a_{i} \in \boldsymbol{Z} .
$$

Now, we assert that every element $\gamma \in \Gamma$ can be written uniquely as $\gamma=\sum_{i=1}^{s} c_{i} x_{i}+\sum_{j=1}^{t+1} d_{j} u_{j}, \quad c_{i}, d_{j} \in \boldsymbol{Z}$ for $i=1, \cdots, s ; j, \cdots t+1$. In fact $\phi(\gamma)=\sum_{i=1} c_{i} \phi\left(x_{i}\right)=\phi\left(\sum_{i=1}^{s} c_{i} x_{i}\right)$ for some $c_{i} \in Z$. Hence $\gamma-\sum c_{i} x_{i} \in W \cap \Gamma$. Therefore $\gamma-\Sigma c_{i} x_{i}=\Sigma d_{j} u_{j}$ for some $d_{j} \in \boldsymbol{Z}$ by the assumption for $W$. The 
uniqueness of the expression (8.2) is easily verified. Since $\Gamma$ is a discrete subgroup of $\boldsymbol{C}^{n}$, we see that $x_{1}, \cdots, x_{s}, u_{1}, \cdots, u_{t+1}$ are linearly independent over $\boldsymbol{R}$ (cf. e.g. [6, p. 27]). Hence we see that $s=n+m-t-1$. Put $w_{i}=u_{i}(i \leqq t+1)$ and $w_{i}=x_{i-t-1}(n+m \geqq i>t+1)$. Finally put

$$
w_{i}=\sum_{j=1}^{n} a_{j i} e_{j}+\sum_{k=1}^{m} a_{n+k, j} v_{k}
$$

for $i=1,2, \cdots, n+m$. Since $w_{1}, \cdots, w_{n+m}$ is a system of generators of $\Gamma$ we see that $M=\left(a_{i j}\right) \in \mathrm{GL}(n+m, Z)$ and that $M$ satisfies the condition (8.1). Thus Theorem 8.1 is proved.

Definition 8.2. We shall call $G$ in Theorem 8.1 a nonsingular abelian Lie group of dimension $n$ and of rank $n+m$. The set of all such groups will be denoted by $A(n, m)$. Then, $A(n, 1)$ coincides with $A^{0}(n)$ in Theorem 5.7.

Combining Theorem 8.1 and 6.4 we have proved the following

Corollary 8.3. Any nonsingular abelian Lie group contains no compact complex submanifold of positive dimension.

THEOREM 8.4. Every nonsingular abelian Lie group $G$ is an (H.C)-group.

Proof. Let $G=C^{n} / \Gamma(V)$ with $V \in \mathfrak{M}^{* *}(n, m ; C)$. If $G$ is not an (H.C)-group, then by Theorem $4.4, G$ is isomorphic to $G_{1} \times C^{*}$ with some subgroup $G_{1}$ of $G$. Then we can suppose that

$$
V=\left(\begin{array}{c}
V_{1} \\
0
\end{array}\right)
$$

with $V_{1} \in \mathfrak{M}^{*}(n-1, m ; C)$. Then by taking

$$
M=\left(e_{1}, \cdots, e_{n-1}, e_{n+1}, e_{n}, e_{n+2}, \cdots, e_{n+m}\right)=\left(\begin{array}{ll}
A & C \\
B & D
\end{array}\right) \in \mathrm{GL}(n+m, Z),
$$

we see that $\operatorname{det}(A-V B)=0$. Hence $G$ is singular. Thus Theorem 8.4 is proved.

Combining Lemma 2.4, Remark 7.6 and Definition 8.2 we obtain the following

THEOREM 8.5. There exists a natural one-one correspondence between $A(n, m)$ and the quotient space $\mathfrak{M}^{* *}(n, m ; C) / \mathrm{GL}(n+m, Z)$ of $\mathfrak{M}^{* *}(n, m ; C)$ by the operation of $\mathrm{GL}(n+m, Z)$ defined in Definition 7.4.

REMARK 8.6. Unfortunately, the converse of Theorem 8.1 does not hold which causes complications in the classifications of complex abelian Lie groups. As is shown in the sequel, there exist many $V \in \mathfrak{M}^{*}(n, m ; C)$ such that $C^{n} / \Gamma(V)$ contains no complex torus and that $V$ is nevertheless singular.

9. Singular (H.C)-groups of dimension 3 and of rank 5. From now on we shall restrict ourselves to the case of dimension 3.

Lemma 9.1. Let $V \in \mathfrak{M}^{*}(3,2 ; C)$. Then $V$ is singular if and only if $C^{3} / \Gamma(V)$ is isomorphic to $C^{3} / \Gamma\left(V^{\prime}\right)$ where $V^{\prime}=\left(v_{1}^{\prime}, v_{2}^{\prime}\right)$ and $v_{2}^{\prime} \in\left\{e_{1}, e_{2}\right\}_{c}$. 
Proof. Suppose $V$ is singular. Then there exists a matrix

$$
M=\left(\begin{array}{ll}
A & C \\
B & D
\end{array}\right) \in \mathrm{GL}(5, Z)
$$

with $A \in \mathfrak{M}(3, Z)$ such that $\operatorname{det}(A+V B)=0$. Put $A+V B=\left(u_{1}, u_{2}, u_{3}\right)$, $C+V D=\left(u_{4}, u_{5}\right)$, where $u_{i} \in C^{3}$ for $i=1,2, \cdots, 5$. We assert that $\operatorname{rank}(A+V B)$ $=2$. In fact, since $u_{1}, u_{2}, u_{3}$ are linearly independent over $\boldsymbol{R}$ (cf. Lemma 2.5), $\operatorname{dim}_{\boldsymbol{c}}\left\{u_{1}, u_{2}, u_{3}\right\}_{\boldsymbol{c}}=\frac{1}{2} \operatorname{dim}_{R}\left\{u_{1}, u_{2}, u_{3}\right\}_{\boldsymbol{c}} \geqq \frac{1}{2} \operatorname{dim}_{R}\left\{u_{1}, u_{2}, u_{3}\right\}_{R}=3 / 2$. Hence $\operatorname{dim}_{c}\left\{u_{1}, u_{2}, u_{3}\right\}_{c}=2$, which means $\operatorname{rank}(A+V B)=2$. Now, since $\operatorname{dim}_{c}\left\{u_{1}, \cdots, u_{5}\right\}_{c}=3$, we can suppose, by changing the indices if necessary, that $u_{1} u_{2}, u_{4}$ are linearly independent over $C$. Take the automorphism $\phi$ of $C^{3}$ such that $\phi\left(u_{1}\right)=e_{1}, \phi\left(u_{2}\right)=e_{2}, \phi\left(u_{4}\right)=e_{3}$ and put $\phi\left(u_{5}\right)=v_{1}^{\prime}, \phi\left(u_{3}\right)=v_{2}^{\prime}$. Then, by using Lemma 2.5 , we see that $C^{3} / \Gamma(V)=C^{3} /\left\{u_{1}, \cdots, u_{5}\right\}_{z} \simeq C^{3} / \Gamma\left(V^{\prime}\right)$ with $V^{\prime}=\left(v_{1}^{\prime}, v_{2}^{\prime}\right)$. Since $u_{3} \in\left\{u_{1}, u_{2}\right\}_{c}$, we see that $v_{2}^{\prime} \in\left\{e_{1}, e_{2}\right\}_{c}$.

To prove the converse it is sufficient to show that $V^{\prime}$ satisfying the condition of Lemma 9.1 is singular. For, if $V$ is nonsingular, $V^{\prime}$ is also nonsingular by Lemma 7.3. Now, if we take

$$
M=\left(e_{5}, e_{2}, e_{1}, e_{3}, e_{4}\right)=\left(\begin{array}{ll}
A & C \\
B & D
\end{array}\right) \in \mathrm{GL}(5, Z) \text { with } A=\left[\begin{array}{lll}
0 & 0 & 1 \\
0 & 1 & 0 \\
0 & 0 & 0
\end{array}\right),
$$

then it is clear that $\operatorname{det}\left(A+V^{\prime} B\right)=0$. Thus Lemma 9.1 is proved.

Definition 9.2. For $V \in \mathfrak{M}^{*}(3,2 ; C)$ and for

$$
M=\left(\begin{array}{ll}
A & C \\
B & D
\end{array}\right) \in \mathrm{GL}(5, Z)
$$

with $A \in \mathfrak{M}(3, Z)$ we define $\chi(V, M) \in Z$ by

$$
\chi(V, M)=\operatorname{rank}(A V-C) .
$$

We shall denote by $\chi(V)$ the minimum of $\chi(V, M)$ for $M \in \mathrm{GL}(5, Z)$. Clearly $0 \leqq \chi(V) \leqq 2$ holds.

Lemma 9.3. Let $V \in \mathfrak{M}^{*}(3,2 ; C)$ be singular and let $C^{3} / \Gamma(V)$ be an (H.C)group. Then, $C^{3} / \Gamma(V) \simeq C^{3} / \Gamma\left(V^{\prime}\right)$ with $V^{\prime}=\left(v_{1}^{\prime}, v_{2}^{\prime}\right), v_{2}^{\prime} \in C e_{1}$, if and only if $\chi(V)=1$.

Proof. We can suppose that $V=\left(v_{1}, v_{2}\right)$ with $v_{2} \in\left\{e_{1}, e_{2}\right\}_{c}$ by Lemma 9.1. Suppose that $\chi(V)=1$. Then there exists

$$
M^{\prime}=\left(\begin{array}{ll}
A^{\prime} & C^{\prime} \\
B^{\prime} & D^{\prime}
\end{array}\right) \in \mathrm{GL}(5, Z)
$$

with $A^{\prime} \in \mathfrak{M}\left(3, Z^{\prime}\right)$ such that $\chi\left(V, M^{\prime}\right)=1$. Put $M^{\prime}=\left(A^{\prime \prime}, C^{\prime \prime}\right)$ with $A^{\prime \prime} \in \mathfrak{M}(5,3 ; Z)$. 
Then

$$
M^{\prime} \cdot\left(\begin{array}{c}
V \\
-E_{2}
\end{array}\right)=A^{\prime \prime} V-C^{\prime \prime}
$$

Since

$$
\operatorname{rank} M^{\prime}\left(\begin{array}{c}
V \\
-E_{2}
\end{array}\right)=\operatorname{rank}\left(\begin{array}{c}
V \\
-E_{2}
\end{array}\right)=2,
$$

we have rank $\left(A^{\prime \prime} V-C^{\prime \prime}\right)=2$. Therefore, we can suppose, by exchanging the rows of $M^{\prime}$ if necessary, that there exists $M \in \mathrm{GL}(5, Z)$ such that

$$
M=\left[\begin{array}{ll}
a_{1} & c_{1} \\
A_{1} & C_{1} \\
a_{2} & c_{2}
\end{array}\right]=\left[\begin{array}{ll}
a_{1} & c_{1} \\
& \\
A_{2} & C_{2}
\end{array}\right]
$$

with $A_{1} \in \mathfrak{M}(3, Z), A_{2} \in \mathfrak{M}(4,3 ; ! Z)$ and $a_{1}, a_{2} \in \mathfrak{M}(1,3 ; Z)$ satisfy the following conditions:

$$
\begin{array}{ll}
\operatorname{rank}\left(A_{2} V-C_{2}\right) & =2, \\
\operatorname{rank}\left(A_{1} V-C_{1}\right) & =1, \\
a_{41} x_{1}+a_{42} y_{1}+a_{43} z_{1}-a_{44} & \neq 0, \text { or } \\
a_{41} x_{2}+a_{42} y_{2}-a_{45} & \neq 0,
\end{array}
$$

where we have put $M=\left(a_{i j}\right)$ and $v_{1}={ }^{t}\left(x_{1}, y_{1}, z_{1}\right)$. If $a_{41} x_{1}+a_{42} y_{1}+a_{43} z_{1}-a_{44}$ $\neq 0$, we can define $y_{1}^{\prime}, z_{1}^{\prime} \in C$ as follows

$$
\begin{aligned}
& y_{1}^{\prime}=\left(a_{24}-a_{21} x_{1}-a_{22} y_{1}-a_{23} z_{1}\right) /\left(a_{41} x_{1}+a_{42} y_{1}+a_{43} z_{1}-a_{44}\right), \\
& z_{1}^{\prime}=\left(a_{34}-a_{31} x_{1}-a_{32} y_{1}-a_{33} z_{1}\right) /\left(a_{41} x_{1}+a_{42} y_{1}+a_{43} z_{1}-a_{44}\right) .
\end{aligned}
$$

Further, we can define $x_{1}^{\prime}, x_{2}^{\prime}$ by the following (9.4), since (9.2) holds:

$$
\begin{gathered}
x_{1}^{\prime}\left(a_{41} x_{1}+a_{42} y_{1}+a_{43} z_{1}-a_{44}\right)+x_{2}^{\prime}\left(a_{51} x_{1}+a_{52} y_{1}-a_{53} z_{1}-a_{54}\right) \\
=a_{14}-a_{11} x_{1}-a_{12} y_{1}-a_{13} z_{1}, \\
x_{1}^{\prime}\left(a_{41} x_{2}+a_{42} y_{2}-a_{45}\right)+x_{2}^{\prime}\left(a_{51} x_{2}+a_{52} y_{2}-a_{55}\right) \\
=a_{15}-a_{11} x_{2}-a_{12} y_{2} .
\end{gathered}
$$

Putting

$$
M=\left(\begin{array}{ll}
A & C \\
B & D
\end{array}\right) \text { with } A \in \mathfrak{M}(3, Z) \text { and } V^{\prime}=\left[\begin{array}{ll}
x_{1}^{\prime} & x_{2}^{\prime} \\
y_{1}^{\prime} & 0 \\
z_{1}^{\prime} & 0
\end{array}\right],
$$

we see, by a simple calculation, that $\left(A+V^{\prime} B\right) V=C+V^{\prime} D$ holds. Hence, by 
Lemma $2.4, C^{3} / \Gamma(V) \simeq C^{3} / \Gamma\left(V^{\prime}\right)$. In the case $a_{41} x_{1}+a_{42} y_{1}+a_{43} z_{1}-a_{44}=0$ and $a_{41} x_{2}+a_{42} y_{2}-a_{45} \neq 0$, we can prove in the same way.

Conversely suppose that $C^{3} / \Gamma(V) \simeq C^{3} / \Gamma\left(V^{\prime}\right)$. Then, by Lemma 2.4 , there exists an $M=\left(a_{i j}\right) \in \mathrm{GL}(5, Z)$ such that $\left(A+V^{\prime} B\right) V=C+V^{\prime} D$ with

$$
M=\left(\begin{array}{ll}
A & C \\
B & D
\end{array}\right)
$$

and $A \in \mathfrak{M}(3, Z)$. This equality implies

$$
\begin{array}{ll}
y_{1}^{\prime}\left(a_{41} x_{1}+a_{42} y_{1}+a_{43} z_{1}-a_{44}\right) & =a_{24}-a_{21} x_{1}-a_{22} y_{1}-a_{23} z_{1}, \\
y_{1}^{\prime}\left(a_{41} x_{2}+a_{42} y_{2}-a_{45}\right) & =a_{25}-a_{21} x_{2}-a_{22} y_{2}, \\
z_{1}^{\prime}\left(a_{41} x_{1}+a_{42} y_{1}+a_{43} z_{1}-a_{44}\right) & =a_{34}-a_{31} x_{1}-a_{32} y_{1}-a_{33} z_{1}, \\
z_{1}^{\prime}\left(a_{41} x_{2}-a_{42} y_{2}-a_{45}\right) & =a_{35}-a_{31} x_{2}-a_{32} y_{2} .
\end{array}
$$

If we put

$$
M=\left[\begin{array}{ll}
a_{1} & c_{1} \\
A_{1} & C_{1} \\
a_{2} & c_{2}
\end{array}\right]
$$

with $a_{1}, a_{2} \in \mathfrak{M}(1,3 ; Z)$ and $A_{1} \in \mathfrak{M}(3, Z)$, then $(9.5)$ mean that $\operatorname{rank}\left(A_{1} V-C_{1}\right) \leqq 1$. Put

$$
M^{\prime}=\left[\begin{array}{ll}
A_{1} & C_{1} \\
a_{1} & c_{1} \\
a_{2} & c_{2}
\end{array}\right]
$$

then we have $\chi\left(V, M^{\prime}\right) \leqq 1$. On the other hand, since $C^{3} / \Gamma(V)$ is an (H.C)-group, $\chi\left(V, M^{\prime \prime}\right) \geqq 1$ for any $M^{\prime \prime} \in \operatorname{GL}(5, Z)$ by Theorem 4.4 . Hence we have $\chi(V)=1$. Thus Lemma 9.3 is proved.

Lemma 9.4. Let $G=C^{3} / \Gamma(V)$ with $V \in \mathfrak{M}^{*}(3,2 ; C)$. Then $G$ contains a 1-dimensional complex torus if and only if $G \simeq C^{3} / \Gamma\left(V^{\prime}\right)$ with $V^{\prime}=\left(v_{1}^{\prime}, v_{2}^{\prime}\right), v_{2}^{\prime} \in C e_{1}$.

Proof. Suppose that $C^{3} / \Gamma(V) \simeq C^{3} / \Gamma\left(V^{\prime}\right)$. Let $\phi$ be the natural homomorphism of $C^{3}$ onto $C^{3} / \Gamma\left(V^{\prime}\right)$. Then $\phi\left(C e_{1}\right)$ is compact, since $C e_{1}$ contains $e_{1}$ and $v_{1}^{\prime}$. Hence $G$ contains a 1 -dimensional complex torus.

Conversely, suppose that $G$ contains a 1-dimensional complex torus $T$. Let $\psi$ be the natural homomorphism of $C^{3}$ onto $G$. Let $K$ be the maximal compact subgroup of $G$ and $\mathfrak{f}(\mathfrak{g}, \mathfrak{t}$ resp. $)$ the Lie algebra of $K\left(G, T\right.$ resp.). Put $\mathfrak{f}_{0}=\mathfrak{f} \cap \sqrt{ }(-1) \mathfrak{f}$. Then clearly $\mathrm{t} \subset \mathfrak{f}_{0}$. Identifying $\mathrm{g}$ with $C^{3}$ we see that $\mathrm{t}$ contains $u_{1}, u_{2} \in \Gamma$ such that $u_{1}=z u_{2} \neq 0$ with $z \in C-\boldsymbol{R}$. We can suppose that $\left\{u_{1}, u_{2}\right\}_{\boldsymbol{R}} \cap \Gamma=\left\{u_{1}, u_{2}\right\}_{\mathbf{z}}$. Then, by the same argument as in the proof of Theorem 8.1, we can find three elements $u_{3}, u_{4}, u_{5} \in \Gamma$ such that $\Gamma=\left\{u_{1}, u_{2}, \cdots, u_{5}\right\}_{z}$. Moreover, we can suppose that $u_{1}, u_{3}, u_{4}$ are linearly independent over $C$. Then we can take an automor- 
phism $\eta$ of $C^{3}$ such that $\eta\left(u_{1}\right)=e_{1}, \eta\left(u_{3}\right)=e_{2}, \eta\left(u_{4}\right)=e_{3}$. Put $\eta\left(u_{5}\right)=v_{1}^{\prime}$, $\eta\left(u_{2}\right)=v_{2}^{\prime}$. Then $C^{3} / \Gamma(V) \simeq C^{3} / \Gamma\left(V^{\prime}\right)$ and $v_{2}^{\prime} \in C e_{1}$. Thus Lemma 9.4 is proved.

Definition 9.5. We denote by $\mathfrak{M}^{\prime}$ the set of all $V \in \mathfrak{M}^{*}(3,2 ; C)$ such that $\chi(C)=2, V=\left(v_{1}, v_{2}\right)$ with $v_{2} \in\left\{e_{1}, e_{2}\right\}_{c}$ and that (4.12) holds for $n=3, m=2$. Let

$$
V=\left[\begin{array}{cc}
x_{1} & x_{2} \\
y_{1} & y_{2} \\
z_{1} & 0
\end{array}\right]
$$

Then it is straightforward to see that, if nine numbers

$$
1, x_{1}, y_{1}, z_{1}, x_{2}, y_{2}, x_{1} y_{2}-x_{2} y_{1}, x_{2} z_{1}, y_{2} z_{1}
$$

are linearly independent over $\boldsymbol{Q}$ then $V \in \mathfrak{M}^{\prime}$.

Combining Lemma 9.1, 9.3 and 9.4 we obtain

Theorem 9.6. Let $V \in \mathfrak{M}^{*}(3,2 ; C)$. Put $G=C^{3} / \Gamma(V)$ and suppose that $G$ is an (H.C)-group. Then, $G$ is singular and contains no complex torus of 1-dimension if and only if $V \in \mathfrak{M}^{\prime}$.

10. Classification of singular (H.C)-groups. I.

Definition 10.1. Let $G$ be a group and $S$ be a set. Suppose that for any $x \in S$ there is given a subset $G_{x}$ of $G$ containing the unit element $e$ and that for any element $g \in G_{x}, g(x) \in S$ is defined. The family $\left\{G_{x}\right\}$ will be called a pseudo-group operating on $S$ if the following three conditions are satisfied:

$$
\begin{aligned}
& e(x)=x \text { for any } x \in S, \\
& g \in G_{x} \text { implies } g^{-1} \in G_{g(x)},
\end{aligned}
$$

$$
g \in G_{x}, g^{\prime} \in G_{g(x)} \text { imply } g^{\prime} g \in G_{x} \text { and }\left(g^{\prime} g\right)(x)=g^{\prime}(g(x)) \text { holds. }
$$

If $\left\{G_{x}\right\}$ is a pseudo-group operating on $S$, we say two elements $x, y \in S$ are equivalent if there exists an element $g \in G_{x}$ such that $g(x)=y$. The set of equivalence classes will be called the quotient space of $S$ by the pseudo-group $\left\{G_{x}\right\}$ and denoted by $S /\left\{G_{x}\right\}$.

DEFINITION 10.2. For $V \in \mathfrak{M}(3,2 ; C)$ and

$$
M=\left[\begin{array}{c}
a_{1} \\
\vdots \\
a_{5}
\end{array}\right] \in \mathrm{GL}(5, Z)
$$

with ${ }^{t} a_{i} \in Z^{5}$, we define $F_{i j}(V, M) \in C$ as follows:

$$
F_{i j}(V, M)=\operatorname{det}\left(\left(\begin{array}{l}
a_{i} \\
a_{j}
\end{array}\right)\left(\begin{array}{c}
V \\
-E_{2}
\end{array}\right)\right)
$$

for $1 \leqq i, j \leqq 5$. 
Definition 10.3. We denote $\Delta=\operatorname{GL}(5, Z)$. For $V \in \mathfrak{M}(3,2 ; C)$ we shall denote by $\Delta_{V}$ the set of all $M \in \Delta$ such that

$$
F_{34}(V, M)=0 .
$$

Clearly $E_{5} \in \Delta_{V}$ for any $V$.

LEMma 10.4. For $V \in \mathfrak{M}^{\prime}$ and for

$$
M=\left(\begin{array}{ll}
A & C \\
B & D
\end{array}\right) \in \Delta_{V}
$$

with $A \in \mathfrak{M}(3, Z)$, there exists uniquely an element $V^{\prime} \in \mathfrak{M}^{\prime}$ such that

$$
\left(A+V^{\prime} B\right) V=C+V^{\prime} D \text {. }
$$

Proof. Put

$$
V=\left[\begin{array}{ll}
x_{1} & x_{2} \\
y_{1} & y_{2} \\
z_{1} & 0
\end{array}\right]
$$

Since $F_{33}(V, M)=0$ and since (4.12) holds for $V$, there exists a unique $z_{1}^{\prime} \in C$ satisfying

$$
\begin{array}{ll}
z_{1}^{\prime}\left(a_{41} x_{1}+a_{42} y_{1}+a_{43} z_{1}-a_{44}\right) & =a_{34}-a_{31} z_{1}-a_{32} y_{1}-a_{33} z_{1}, \\
z_{1}^{\prime}\left(a_{41} x_{2}+a_{42} y_{2}-a_{45}\right) & =a_{35}-a_{31} x_{2}-a_{32} z_{2} .
\end{array}
$$

Next, since $\chi(V)=2$, we see that $F_{45}(V, M) \neq 0$. Hence we can define $x_{1}^{\prime}, x_{2}^{\prime}, y_{1}^{\prime}$ and $y_{2}^{\prime} \in C$ by the following equalities:

$$
\begin{aligned}
& x_{1}^{\prime}=F_{51}(V, M) / F_{45}(V, M), x_{2}^{\prime}=F_{41}(V, M) / F_{45}(V, M), \\
& y_{1}^{\prime}=F_{52}(V, M) / F_{45}(V, M), y_{2}^{\prime}=F_{42}(V, M) / F_{45}(V, M)
\end{aligned}
$$

We put

$$
V^{\prime}=\left[\begin{array}{ll}
x_{1}^{\prime} & x_{2}^{\prime} \\
y_{1}^{\prime} & y_{2}^{\prime} \\
z_{1}^{\prime} & 0
\end{array}\right]
$$

By a direct calculation we see that (10.5), (10.6) imply the following equality

$$
\left(A+V^{\prime} B\right) V=C+V^{\prime} D \text {. }
$$

Hence, by Lemma $2.4, C^{3} / \Gamma(V) \simeq C^{3} / \Gamma\left(V^{\prime}\right)$. Since $C^{3} / \Gamma(V)$ contains no complex torus of dimension $1, C^{3} / \Gamma\left(V^{\prime}\right)$ contains no complex torus of dimension 1 . Therefore, by Theorems 9.6 and 4.4, $V^{\prime} \in \mathfrak{M}^{\prime}$. Now, by Lemma 2.3, (10.7) implies $\left(A^{\prime}+V B^{\prime}\right) V^{\prime}=C^{\prime}+V D^{\prime}$, where

$$
M^{-1}=\left(\begin{array}{ll}
A^{\prime} & C^{\prime} \\
B^{\prime} & D^{\prime}
\end{array}\right)
$$


with $A^{\prime} \in \mathfrak{M}(3, Z)$. Then, by Lemma 2.2 , $\operatorname{det}\left(A^{\prime}+V B^{\prime}\right) \neq 0$, and so $V^{\prime}=\left(A^{\prime}+V B^{\prime}\right)^{-1} \cdot\left(C^{\prime}+V D^{\prime}\right)$, which proves the uniqueness of $V^{\prime}$. Thus Lemma 10.4 is proved.

Definition 10.5. For $V \in \mathfrak{M}^{\prime}$ and $M \in \Delta_{V}$, the element $V^{\prime}$ in Lemma 10.4 will be denoted by $V^{\prime}=M(V)$.

Lemma 10.6. Let $M \in \Delta$ and $V \in \mathfrak{M}^{\prime}$. Then $M \in \Delta_{V}$ if and only if there exists $V^{\prime} \in \mathfrak{M}(3,2 ; C)$ such that $(10.7)$ holds, where we put

with $A \in \mathfrak{M}(3, Z)$.

$$
M=\left(\begin{array}{ll}
A & C \\
B & D
\end{array}\right)
$$

Proof. Put

$$
V=\left[\begin{array}{ll}
x_{1} & x_{2} \\
y_{1} & y_{2} \\
z_{1} & 0
\end{array}\right] \text { and } V^{\prime}=\left[\begin{array}{ll}
x_{1}^{\prime} & x_{2}^{\prime} \\
y_{1}^{\prime} & y_{2}^{\prime} \\
z_{1}^{\prime} & 0
\end{array}\right]
$$

By a simple calculation, (10.7) implies the equalities (10.5). We assert that $z_{1}^{\prime} \neq 0$. In fact, if $z_{1}^{\prime}=0$, we see that $C^{3} / \Gamma(V) \simeq T \times C^{*}$, where $T$ is a complex torus of dimension 2 , which is a contradiction. Then, since $z_{1}^{\prime} \neq 0,(10.5)$ implies $F_{34}(V, M)=0$. Hence $M \in \Delta_{V}$. The converse is already proved in Lemma 10.4 . Thus Lemma 10.6 is proved.

THEOREM 10.7. The family $\left\{\Delta_{V}\right\}, V \in \mathfrak{M}^{\prime}$ is a pseudo-group operating on $\mathfrak{M}^{\prime}$.

Proof. First, for $V \in \mathfrak{M}^{\prime}$ and $M \in \Delta_{V}, V^{\prime}=M(V)$ is well defined by Lemma 10.4. The condition (10.1) is trivial. We shall prove (10.3) for $\left\{\Delta_{V}\right\}$. Take $V \in M^{\prime}$, $M \in \Delta_{V}$ and $M^{\prime} \in \Delta_{M(V)}$. Put $V^{\prime}=M(V)$ and $V^{\prime \prime}=M^{\prime}(M(V))$, and put

$$
M=\left(\begin{array}{ll}
A & C \\
B & D
\end{array}\right), M^{\prime}=\left(\begin{array}{ll}
A^{\prime} & C^{\prime} \\
B^{\prime} & D^{\prime}
\end{array}\right), M^{\prime} M=\left(\begin{array}{ll}
A^{\prime \prime} & C^{\prime \prime} \\
B^{\prime \prime} & D^{\prime \prime}
\end{array}\right)
$$

with $A, A^{\prime}, A^{\prime \prime} \in \mathfrak{M}(3, Z)$. Then, we have the following two equalities

$$
\begin{gathered}
\left(A+V^{\prime} B\right) V=C+V^{\prime} D, \\
\left(A^{\prime}+V^{\prime \prime} B^{\prime}\right) V^{\prime}=C^{\prime}+V^{\prime \prime} D^{\prime},
\end{gathered}
$$

which imply $\left(A^{\prime \prime}+V^{\prime \prime} B^{\prime \prime}\right) V=C^{\prime \prime}+V^{\prime \prime} D^{\prime \prime}$, which shows, by Lemmas 10.4 and 10.6, that $M^{\prime} M \in \Delta_{V}$ and that $V^{\prime \prime}=\left(M^{\prime} M\right)(V)$. Hence we obtain $M^{\prime}(M(V))=\left(M^{\prime} M\right)(V)$ which proves (10.3).

Next, we shall prove (10.2). For this purpose we put $V^{\prime}=M(V)$ and

$$
M^{-1}=\left(\begin{array}{ll}
A^{\prime} & C^{\prime} \\
B^{\prime} & D^{\prime}
\end{array}\right)
$$

with $A^{\prime} \in \mathfrak{M}(3, Z)$. Then we have $\left(A+V^{\prime} B\right) V=C+V^{\prime} D$, which implies, by 
Lemma 2.3, $\left(A^{\prime}+V B^{\prime}\right) V^{\prime}=C^{\prime}+V D^{\prime}$, which shows, by Lemma 10.6, that $M^{-1} \in \Delta_{V^{\prime}}=\Delta_{M(V)}$ and $V=M^{-1}\left(V^{\prime}\right)$. Thus Theorem 10.7 is proved.

From the above considerations we have proved the following

THEOREM 10.8. Let $\boldsymbol{A}^{\prime}(3,2)$ be the set of all singular (H.C)-groups of dimension 3 and of rank 5 which contains no complex torus of dimension 1. Then there exists a natural one-one correspondence between $A^{\prime}(3,2)$ and the quotient space $M^{\prime} /\left\{\Delta_{V}\right\}$.

11. Classification of singular (H.C)-group. II.

Definition 11.1. We denote by $\mathfrak{M}^{\prime \prime}$ the set of all $V \in \mathfrak{M}^{*}(3,2 ; C)$ such that $V=\left(v_{1}, v_{2}\right)$ and $v_{2} \in C e_{1}$ and that $V$ satisfies (4.12) for $n=3, n=2$. For $M \in \Delta=\mathrm{GL}(5, Z)$, we put

$$
M \cdot\left(\begin{array}{c}
V \\
-E_{2}
\end{array}\right)=\left[\begin{array}{c}
a_{1} \\
\vdots \\
a_{5}
\end{array}\right]
$$

with $a_{t} \in C^{2}(i=1,2, \cdots, 5)$. We say $a_{i}$ and $a_{j}$ are equivalent if $C a_{l}=C a_{j}$ and we denote by $v(V, M)$ the number of equivalence classes of $a_{1}, \cdots, a_{5}$ by this equivalence relation. Clearly $2 \leqq v(V, M) \leqq 5$. We shall denote by $v(V)$ the minimum of $v(V, M)$ for $M \in \Delta$. Then it is easily seen that $2 \leqq v(V) \leqq 3$.

Let

$$
V=\left[\begin{array}{ll}
x_{1} & x_{2} \\
y_{1} & 0 \\
z_{1} & 0
\end{array}\right]
$$

We can easily see that, if seven numbers $1, x_{1}, y_{1}, z_{1}, x_{2}, x_{2} y_{1}, x_{2} z_{1}$ are linearly independent over $Q, v(V)=3$.

LEMMA 11.2. Take $V \in \mathfrak{M}^{\prime \prime}$. Then the group $G=C^{3} / \Gamma(V)$ contains a 1-dimensional complex torus as a direct summand if and only if $v(V)=2$.

Proof. Suppose $v(V, M)=2$ for some $M=\left(a_{i j}\right) \in \Delta$. We can suppose, exchanging the indices if necessary, that $F_{45}(V, M) \neq 0$. We assert that $F_{24}(V, M)=F_{34}(V, M)=F_{15}(V, M)=0$. If not, we may assume that $F_{14}(V, M)=F_{24}(V, M)=F_{34}(V, M)=0$. In this case, putting

$$
V=\left[\begin{array}{ll}
x_{1} & x_{2} \\
y_{1} & 0 \\
z_{1} & 0
\end{array}\right],
$$

we can define $x_{1}^{\prime}, y_{1}^{\prime}, z_{1}^{\prime} \in C$ by the following equalities:

$$
\begin{aligned}
x_{1}^{\prime}\left(a_{41} x_{1}+a_{42} y_{1}+a_{43} z_{1}-a_{44}\right) & =a_{14}-a_{11} x_{1}-a_{12} y_{1}-a_{13} z_{1}, \\
x_{1}^{\prime}\left(a_{41} x_{2}-a_{45}\right) & =a_{15}-a_{11} x_{2},
\end{aligned}
$$




$$
\begin{array}{ll}
y_{1}^{\prime}\left(a_{41} x_{1}+a_{42} y_{1}+a_{43} z_{1}-a_{44}\right) & =a_{24}-a_{21} x_{1}-a_{22} y_{1}-a_{23} z_{1}, \\
y_{1}^{\prime}\left(a_{41} x_{2}-a_{45}\right) & =a_{25}-a_{21} x_{2}, \\
z_{1}^{\prime}\left(a_{41} x_{1}+a_{42} y_{1}+a_{43} z_{1}-a_{44}\right) & =a_{34}-a_{31} x_{1}-a_{32} y_{1}-a_{33} z_{1}, \\
z_{1}^{\prime}\left(a_{41} x_{2}-a_{45}\right) & =a_{35}-a_{31} x_{2} .
\end{array}
$$

Put

$$
V^{\prime}=\left[\begin{array}{ll}
x_{1}^{\prime} & 0 \\
y_{1}^{\prime} & 0 \\
z_{1}^{\prime} & 0
\end{array}\right] \in \mathfrak{M}(3,2 ; C) \text { and } M=\left(\begin{array}{cc}
A & C \\
B & D
\end{array}\right)
$$

with $A \in \mathfrak{M}(3, Z)$. Then (11.1)-(11.6) show that $\left(A+V^{\prime} B\right) V=C+V^{\prime} D$ holds. Hence, by Lemmas 2.2 and 2.3 , we have $V^{\prime} \in \mathfrak{M}^{*}(3,2 ; Z)$, which is a contradiction. Thus our assertion is verified.

Since $F_{24}(V, M)=F_{34}(V, M)=F_{15}(V, M)=0$, we can define $x_{2}^{\prime}, y_{1}^{\prime}, z_{1}^{\prime} \in C$ by the following equalities:

$$
\begin{aligned}
& x_{2}^{\prime}\left(a_{51} x_{1}+a_{52} y_{1}+a_{53} z_{1}-a_{54}\right)=a_{14}-a_{11} x_{1}-a_{12} y_{1}-a_{13} z_{1}, \\
& x_{2}^{\prime}\left(a_{51} x_{2}-a_{55}\right)=a_{15}-a_{11} x_{2} \text {, } \\
& y_{1}^{\prime}\left(a_{41} x_{1}+a_{42} y_{1}+a_{43} z_{1}-a_{44}\right)=a_{24}-a_{21} x_{1}-a_{22} y_{1}-a_{23} z_{1}, \\
& \text { (11.10) } y_{1}^{\prime}\left(a_{41} x_{2}-a_{45}\right) \\
& =a_{25}-a_{21} x_{2}, \\
& z_{1}^{\prime}\left(a_{41} x_{1}+a_{42} y_{1}+a_{43} z_{1}-a_{44}\right)=a_{34}-a_{31} x_{1}-a_{32} y_{1}-a_{33} z_{1}, \\
& =a_{35}-a_{31} x_{2} \text {. }
\end{aligned}
$$$$
\text { (11.12) } z_{1}^{\prime}\left(a_{41} x_{2}-a_{45}\right)
$$

Putting

$$
V^{\prime}=\left[\begin{array}{ll}
0 & x_{2}^{\prime} \\
y_{1}^{\prime} & 0 \\
z_{1} & 0
\end{array}\right]
$$

we see that (11.7)-(11.12) are equivalent to the equality $\left(A+V^{\prime} B\right) V=C+V^{\prime} D$ for

$$
M=\left(\begin{array}{ll}
A & C \\
B & D
\end{array}\right)
$$

with $A \in \mathfrak{M}(3, Z)$. Put $v^{\prime}=\left(y_{1}^{\prime}, z_{1}^{\prime}\right)$. Then by Lemma 2.4,

$$
G \simeq C^{1} /\left\{1, x_{2}^{\prime}\right\}_{\mathrm{Z}} \times C^{2} / \Gamma\left(v^{\prime}\right)=T \times C^{2} / \Gamma\left(v^{\prime}\right),
$$

where $T$ is a 1-dimensional complex torus.

Conversely, if $G \cong T \times G_{1}$, where $T$ is a 1-dimensional complex torus, the 1 it is easy to find $x_{2}^{\prime}, y_{1}, z_{1}^{\prime} \in C$ and $M=\left(a_{i j}\right)$ satisfying the equalities (11.7) $\sim(11.12)$ Then $v(V, M)=2$, whence $v(V)=2$. Thus Lemma 11.2 is proved.

Definition 11.3. We denote by $\mathfrak{M}^{m}$ the set of all $V \in \mathfrak{M}^{*}\left(3,2 ; C^{*}\right)$ such that $V=\left(v_{1}, v_{2}\right), v_{2} \in C e_{1}, v(V)=3$ and that $V$ satisfies the condition (4.12) for 
$n=3, m=2$. For $V \in \mathfrak{M}^{m}$, we denote by $\Delta^{\prime}{ }_{V}$ the set of all $M \in \Delta$ such that $F_{42}(V, M)=F_{43}(V, M)=0$.

LEMMA 11.4. For any $V \in \mathfrak{M}^{m}$ and $M \in \Delta^{\prime}{ }_{V}$ we can find uniquely an element $V^{\prime} \in \mathfrak{M}^{\prime \prime \prime}$ such that $\left(A+V^{\prime} B\right) V=C+V^{\prime} D$, where

$$
M=\left(\begin{array}{ll}
A & C \\
B & D
\end{array}\right)
$$

with $A \in M(3, Z)$. If we denote $V^{\prime}=M(V)$, then the family $\left\{\Delta_{V}^{\prime}\right\}$ is a pseduogroup operating on $M^{m}$.

In fact, we can prove this lemma exactly in the same manner as the proof of Theorem 10.7. We may omit the proof in detail.

From the above considerations we have proved the following.

THEOREM 11.5. Let $A^{\prime \prime}(3,2)$ be the set of all singular (H.C)-groups of dimension 3 and of rank 5 which contains a 1-dimensional complex torus not as a direct summand. Then there exists a natural one-one correspondence between $A^{\prime \prime}(3,2)$ and the quotient space $\mathfrak{M}^{m} /\left\{\Delta_{V}^{\prime}\right\}$.

12. Final remarks. Summarizing the results obtained in the preceding sections, we obtain the complete classification of noncompact connected complex abelian Lie groups of dimension 3.

We denote by $\tilde{A}(n)$ the set of all connected complex abelian Lie groups of dimension $n$, two isomorphic ones being of course identified. We denote by $\tilde{\boldsymbol{A}}^{0}(n)$, $\tilde{A}^{\prime}(n), \boldsymbol{A}^{0}(n)$ and $\boldsymbol{T}(n)$ the set of all noncompact (H.C)-groups of dimension $n$, non-(H.C)-groups of dimension $n$, (H.C)-groups of dimension $n$ and of rank $n+1$, and the complex tori of dimension $n$ respectively. Let $A(3)$ be the set of all nonsingular groups of dimension 3 and of rank 5. Let $\boldsymbol{A}^{\prime}(3)$ be the set of all singular (H.C)-groups of dimension 3 and of rank 5 containing no complex torus of dimension 1. Finally let $\boldsymbol{A}^{\prime \prime}(3)$ be the set of all singular (H.C)-groups of dimension 3 and of rank 5 containing a 1-dimensional complex torus not as a direct summand. Then, we have the following

THEOREM 12.1.

$$
\begin{aligned}
\tilde{A}(3) & =\tilde{A}^{0}(3) \cup \tilde{A}^{\prime}(3) \cup T(3)(\text { disjoint }), \\
\tilde{A}^{0}(3) & =\boldsymbol{A}^{0}(3) \cup A(3) \cup A^{\prime}(3) \cup \boldsymbol{A}^{\prime \prime}(3) \cup \boldsymbol{T}(1) \times \boldsymbol{A}^{0}(2)(\text { disjoint }), \\
\boldsymbol{A}^{0}(n) & =\mathfrak{M}^{* *}(n, 1 ; C) / \mathrm{GL}(n+1, Z), \\
\boldsymbol{A}(3) & =\mathfrak{M}^{* *}(3,2 ; C) / \mathrm{GL}(5, Z), \\
\boldsymbol{A}^{\prime}(3) & =\mathfrak{M}^{\prime} /\left\{\Delta_{V}\right\} \\
\boldsymbol{A}^{\prime \prime}(3) & =\mathfrak{M}^{m} /\left\{\Delta_{V}^{\prime}\right\} \\
\tilde{A}^{\prime}(3) & =\left\{C, C^{*}\right\} \times \tilde{A}(2)
\end{aligned}
$$


REMARK 12.2. Any one of the subsets $A^{0}(3), A(3), A^{\prime}(3)$ or $A^{\prime \prime}(3)$ contains noncountably many elements.

REMARK 12.3. For any (H.C)-group $G$ the group of holomorphic homeomorphisms of $G$ is a complex Lie group, whose Lie algebra is isomorphic to the one of $G$.

\section{REFERENCES}

1. C. Chevalley, Theory of Lie groups. I, Princeton Univ. Press, Princeton, N. J., 1946.

2. K. Kopfermann, Maximale Untergruppen Abelscher komplexer Liescher Gruppen, Schr. Math. Inst. Univ. Münster No. 29 (1964).

3. P. Lelong, Les fonctions plurisousharmoniques, Ann. École Norm. Sup. 62 (1945),301-338.

4. Y. Matsushima and A. Morimoto, Sur certains espaces fibrés holomorphes sur une variété de Stein, Bull. Soc. Math. France 88 (1960), 137-155.

5. A. Morimoto, Non-compact complex Lie groups without non-constant holomorphic functions, Proc. Conference on Complex Analysis at Univ. of Minn., Springer-Verlag, Berlin, 1965 ; pp. 257-272.

6. C. L. Siegel, Analytic functions of several complex variables, Institute for Advanced Study, Princeton, N. J., 1948-1949.

NAGOYA UNIVERSITY, NAGOYA, JAPAN

University of California at San Diego, la Jolla, California 